\title{
Zero-sum games with charges
}

Citation for published version (APA):

Flesch, J., Vermeulen, D., \& Zseleva, A. (2017). Zero-sum games with charges. Games and Economic Behavior, 102, 666-686. https://doi.org/10.1016/j.geb.2016.10.014

Document status and date:

Published: 01/03/2017

DOI:

10.1016/j.geb.2016.10.014

Document Version:

Publisher's PDF, also known as Version of record

Document license:

Taverne

Please check the document version of this publication:

- A submitted manuscript is the version of the article upon submission and before peer-review. There can be important differences between the submitted version and the official published version of record.

People interested in the research are advised to contact the author for the final version of the publication, or visit the DOI to the publisher's website.

- The final author version and the galley proof are versions of the publication after peer review.

- The final published version features the final layout of the paper including the volume, issue and page numbers.

Link to publication

\footnotetext{
General rights rights.

- You may freely distribute the URL identifying the publication in the public portal. please follow below link for the End User Agreement:

www.umlib.nl/taverne-license

Take down policy

If you believe that this document breaches copyright please contact us at:

repository@maastrichtuniversity.nl

providing details and we will investigate your claim.
}

Copyright and moral rights for the publications made accessible in the public portal are retained by the authors and/or other copyright owners and it is a condition of accessing publications that users recognise and abide by the legal requirements associated with these

- Users may download and print one copy of any publication from the public portal for the purpose of private study or research.

- You may not further distribute the material or use it for any profit-making activity or commercial gain

If the publication is distributed under the terms of Article $25 \mathrm{fa}$ of the Dutch Copyright Act, indicated by the "Taverne" license above, 


\title{
Zero-sum games with charges
}

\author{
János Flesch, Dries Vermeulen*, Anna Zseleva \\ Maastricht University, School of Business and Economics, Dept. of Quantitative Economics, P.O. Box 616, 6200 MD Maastricht, The Netherlands
}

\section{A R T I C L E I N F O}

Article history:

Received 1 March 2016

Available online 15 November 2016

\section{JEL classification:}

$\mathrm{C} 72$

Keywords:

Infinite games

Two-person zero-sum games

Finitely additive strategies

The Wald game

\begin{abstract}
A B S T R A C T
We consider two-player zero-sum games with infinite action spaces and bounded payoff functions. The players' strategies are finitely additive probability measures, called charges. Since a strategy profile does not always induce a unique expected payoff, we distinguish two extreme attitudes of players. A player is viewed as pessimistic if he always evaluates the range of possible expected payoffs by the worst one, and a player is viewed as optimistic if he always evaluates it by the best one. This approach results in a definition of a pessimistic and an optimistic guarantee level for each player. We provide an extensive analysis of the relation between these guarantee levels, and connect them to the classical guarantee levels, and to other known techniques to define expected payoffs, based on computation of double integrals. In addition, we also examine existence of optimal strategies with respect to these guarantee levels.
\end{abstract}

(C) 2016 Elsevier Inc. All rights reserved.

\section{Introduction}

The theory of zero-sum games starts with von Neumann (1928), who showed that zero-sum games with finite action spaces admit a value. Games with infinite action spaces are much more complex, and Wald (1945) demonstrated that such games do not always have a value.

When the action spaces are infinite, the definition of mixed strategies is not trivial. In game theory the usual approach is to define mixed strategies as countably additive probabilities on the actions. A notable, but less frequent alternative is to define mixed strategies as finitely additive probabilities, so-called charges. Since finite additivity is a weaker requirement than countable additivity, the latter approach allows for a richer class of mixed strategies. Charges have regularly been argued for from a conceptual point of view, but they also provide technical advantages.

In this paper, we are interested in the best expected payoff that a charge can guarantee for a player. Since the expected payoff can be defined in various manners, we obtain several guarantee levels. Our goal is to examine these guarantee levels in detail, and to find conditions under which the value of a game exists.

Our setup We study general two-player zero-sum games with infinite, possibly uncountable, action spaces and bounded payoff functions. The strategy space of a player is the set of probability charges defined on all subsets of that player's action

\footnotetext{
We would like to thank Valerio Capraro, Ferenc Forgó, Ehud Lehrer, Christian Nauerz, Miklós Pintér, Marco Scarsini, and William Sudderth for their helpful comments and discussion. We also thank the Dutch institute NWO (Grant no. 040.11.495) for funding the research visit of William Sudderth. We would also like to thank the editor and two anonymous referees.

* Corresponding author.

E-mail addresses: j.flesch@maastrichtuniversity.nl (J. Flesch), d.vermeulen@maastrichtuniversity.nl (D. Vermeulen), a.zseleva@maastrichtuniversity.nl (A. Zseleva).
} 
space. The game is now played as follows. Each player simultaneously selects a probability charge on his action space. These choices are then recorded in a strategy profile. An extension of such a profile is a probability charge that is defined on all subsets of the set of action profiles that coincides on product sets (rectangles) with the product of the strategies.

Given a strategy profile of probability charges, there may be many such extensions. And any extension induces an expected payoff to each player, so that the expected payoff may not be uniquely defined. Since the set of expected payoffs we can thus construct is not necessarily singleton, we study several ways-some of them novel, some of them introduced before-to define the value of the resulting game, or better, class of possible games.

The first two-novel-notions relate to two extreme attitudes of players. In our framework, a player is viewed as pessimistic if from the range of possible expected payoffs he always selects the worst one. Equivalently, he always selects an extension that results in the worst possible payoff. ${ }^{1}$ In contrast, a player is viewed as optimistic if from the range of possible expected payoffs he always selects the best one. This approach results in a definition of a pessimistic and an optimistic guarantee level for each player.

The second pair of notions relate to the two possible ways to compute double integrals with respect to the chosen probability charges. Each double integral assigns an expected payoff to both players. Note that for these notions the choice of extension depends only on the strategy profile, not on the payoff function of the zero-sum game.

Our contribution Starting with a given zero-sum game, endowing each player with one of the above approaches to compute expected payoffs yields a new game. We argue that, given a zero-sum game, the resulting games may all be different. In each such a resulting game, a player can compute the maximum payoff he can guarantee for himself, which leads to a guarantee level for that player. We provide an extensive analysis of the relation between these guarantee levels. In addition, we also examine existence of optimal strategies with respect to these guarantee levels. Our main findings are:

[1] The pessimistic guarantee level for player 1, the optimistic guarantee level for player 2, and the guarantee levels for both players in which we first integrate with respect to the strategy of the 2nd player and then with respect to the 1st player, all coincide. Similarly, the optimistic guarantee level for player 1, the pessimistic guarantee level for player 2 and the guarantee levels arising from the reverse order of integration all coincide as well (cf. Theorem 4.1).

[2] Moreover, the guarantee levels defined above can be calculated by computing so-called finitistic guarantee levels, associated with finite approximations of the resulting games (cf. Theorem 4.1).

[3] Thus, when one player is optimistic, and the other is pessimistic, for any zero-sum game we start with, the resulting (zero-sum) game has a value. The same holds when both players use the same order of integration for the approach based on double integrals: the resulting game is still zero-sum, and the value exists (cf. Corollary 6.1 and 6.2).

This is a novel result in the sense that we establish an existence result for a class of payoff functions that is outside the scope of earlier papers such as Capraro and Scarsini (2013), Harris et al. (2005), Marinacci (1997) and Reny (1999).

A counterexample illustrates that it is not a general fact that the game has a value when the resulting game is zero-sum. We construct a zero-sum game (a game in which the players agree on how to compute expected payoffs) in which the guarantee levels for the players do not coincide. In other words, the resulting zero-sum game does not have a value (cf. Example 6.1).

[4] We prove that players always have optimal strategies with respect to the optimistic guarantee level (cf. Theorem 5.1). We also show that a pessimistic optimal strategy of a player, when it exists, ${ }^{2}$ is also optimal for that player when he computes payoffs by a double integral where the integration is first over strategies of his opponent (cf. Proposition 5.3).

[5] For countable action spaces we show that the classical guarantee level for a player, when players only play countably additive probability distributions, coincides with the pessimistic guarantee level of that player (cf. Theorem 4.3).

\subsection{Related literature}

Probabilities There are different ways of defining probabilities. Countable additivity is a usual assumption of probability. However, from a conceptual point of view, the weaker assumption of finite additivity was also argued for, see de Finetti (1975), Savage (1972), and Dubins et al. (2014). Since finite additivity is the weaker assumption, it is also more basic. It allows for a richer class of probabilities, and facilitates constructions such as a uniform probability distribution over the natural numbers (see Schirokauer and Kadane, 2007). Moreover, translation invariant charges can be defined on all subsets of the real line, and translation and rotation invariant charges can be defined on all subsets of the plane. A notable advantage of finitely additive measures, more briefly called charges, is that they avoid the problem of measure (cf. Aliprantis and Border, 2005). Finally, in decision theory and game theory, only approximate solutions may be achieved with countably additive strategies, while exact solutions can be achieved when using charges (e.g. Sudderth, 2016). For a more extensive summary of the history of finite additivity and its relation to countable additivity, see Bingham (2010).

\footnotetext{
1 Note that this type of selection of the extension crucially depends on the payoffs of the game, not merely on the strategy profile that is chosen.

2 Existence of optimal strategies for pessimistic players is an open question.
} 
Decision theory and game theory In decision theory, charges have been used in various models, notably in de Finetti (1975), Savage (1972) and Dubins et al. (2014). Charges also gained recognition in game theory, such as in Marinacci (1997), Capraro and Scarsini (2013), Harris et al. (2005), and Maitra and Sudderth (1993, 1998), but to a lesser extent. One possible reason is that in a game setting it can occur that multiple players choose actions simultaneously. If each of them uses a probability charge for the choice of his action, then on the space of action profiles, as pointed out above, those choices induce a unique charge only on the field generated by the cylinder sets. This field is fairly restricted, and consequently, there is only a limited class of payoff functions for which the expected payoff is uniquely defined. For instance, Marinacci (1997) and Harris et al. (2005) consider only payoff functions that can be uniformly approximated by simple functions on this field.

In our setup we study a wider range of utility functions, however we consider only games that are zero-sum. As mentioned before, Marinacci (1997) and Harris et al. (2005) consider a restricted class of utility functions where the extension is unique. In this setting they prove the existence of Nash-equilibrium in nonzero-sum games. Capraro and Scarsini (2013) take specific types of extensions, specific classes of payoff functions and also prove existence of Nash-equilibrium in nonzero-sum games. These results indicate that generalization of our results to nonzero-sum games may require a considerable restriction of the set of utility functions.

Relating to our first pair of notions, a well-known definition of pessimism by Wald (1950) is when the decision maker assumes that whichever act he might choose, the worst state will be realized. In our framework, the pessimistic player assumes that from a range of possible expected payoffs the worst one will be realized.

Relating to our second pair of notions, taking a fixed order of integration as the expected payoff has been analyzed before by Heath and Sudderth (1972), and Maitra and Sudderth (1993, 1998). Yanovskaya (1970) and Kindler (1983) consider the double integral as an expected payoff only when different orders of integration yield the same expected payoff. For all other strategy profiles, i.e. when the order of integration does make a difference, they set an arbitrary number as an expected payoff. Schervish and Seidenfeld (1996) take convex combinations of the two orders of integration in zero-sum games, and take those as possible expected payoffs. Capraro and Scarsini (2013) also look at convex combinations of different orders of integration, but they consider countable n-player nonzero-sum games defined through an algebraic operator. We compare their approach to ours through Example 3.4.

As mentioned earlier, when the players agree on how to compute expected payoffs, the resulting game is zero-sum. However, this is not true in general when players disagree on the computation. This is comparable to the model of Aumann (1974), where the players might assign different subjective probabilities to the same event, and consequently, the resulting game might not be zero-sum anymore.

Ambiguity Our pessimistic approach is somewhat related to ambiguity. For example, the maximin expected utility model proposed by Gilboa and Schmeidler (1989) also computes the lowest expected utility over a set of possible probability distributions.

When using charges as strategies in game theory, one of the intriguing questions is how to define an integral over the product of action spaces. A problem of integration also arises when modeling ambiguity through non-additive probabilities, so-called capacities. Schmeidler (1989) made use of the Choquet integral, and Lehrer (2009) defined a concave integral, which can be interpreted as uncertainty aversion.

Structure of the paper Our paper is structured as follows. In Section 2 we discuss some preliminaries. In Section 3 we introduce the zero-sum games we consider and the different ways of calculating expected payoffs. Section 4 contains our solution concepts and main results. Section 5 deals with optimal strategies, and Section 6 is about games where the players consider the same extension.

\section{Preliminaries}

In this section we provide a brief summary on probability charges. For further reading, we refer to Rao and Rao (1983) and Dunford and Schwartz (1964).

Let $X$ be an infinite set. By $\mathcal{P}(X)$ denote the set of all subsets of $X$. The set of all finite subsets of $X$ is denoted by $\mathcal{F}(X)$. A probability charge on $X$ is a function $\mu: \mathcal{P}(X) \rightarrow[0,1]$ such that $\mu(X)=1$ and for all disjoint sets $E, F \in \mathcal{P}(X)$ it holds that $\mu(E \cup F)=\mu(E)+\mu(F){ }^{3}$ We denote the set of all probability charges on $X$ by $\mathcal{C}(X)$, and the set of countably additive probability measures on $X$ by $\Delta(X)$. Thus, $\Delta(X) \subseteq \mathcal{C}(X)$. For $x \in X, \delta(x)$ denotes the Dirac measure on $x$. So, for every set $B \subset X$,

$$
\delta(x)(B)= \begin{cases}1 & \text { if } x \in B, \\ 0 & \text { if } x \notin B .\end{cases}
$$

Note that $\delta(x) \in \Delta(X)$ for every $x \in X$.

\footnotetext{
${ }^{3}$ A probability charge is thus finitely additive, but not necessarily countably additive.
} 
A function $s: X \rightarrow \mathbb{R}$ is called a simple function if there are $c_{1}, \ldots, c_{m} \in \mathbb{R}$ and a partition $\left\{B_{1}, \ldots, B_{m}\right\}$ of $X$ such that $s=\sum_{i=1}^{m} c_{i} \mathbb{I}_{B_{i}}$, where $\mathbb{I}_{B_{i}}$ is the characteristic function of the set $B_{i}$. With respect to a probability charge $\mu$ on $X$, the integral of $s$ is defined by $\int_{X} s d \mu=\sum_{i=1}^{m} c_{i} \cdot \mu\left(B_{i}\right)$.

Let $\mu$ be a probability charge on $X$, and let $f: X \rightarrow \mathbb{R}$ be a bounded function. The integral $\int_{X} f d \mu$ is defined as the supremum of all real numbers $\int_{X} s d \mu$, where $s$ is a simple function and $s \leq f$. Note that, since $f$ is bounded, the integral is finite.

When $X$ is countably infinite, we say that $\mu \in \mathcal{C}(X)$ is a pure probability charge if $\mu(\{x\})=0$ for every $x \in X{ }^{4}$ It follows from Theorem C.3 that pure charges exist. ${ }^{5}$ We denote the set of all pure probability charges on $X$ by $\mathcal{Q}(X)$. Since pure charges are not countably additive, this shows that in this case $\Delta(X)$ is a strict subset of $\mathcal{C}(X)$.

\section{Games with charges}

In this section, we define the model of games with charges.

We focus on two-player zero-sum games. For $i=1,2$, the set $A_{i}$ is the set of actions of player $i$. The sets $A_{i}$ are not empty, and may be, but need not be, infinite. Let $u: A_{1} \times A_{2} \rightarrow \mathbb{R}$ be a bounded payoff function. The zero-sum game $g(u)$ is a game that is played as follows. Player 1 chooses an action $m \in A_{1}$ and independently player 2 chooses an action $n \in A_{2}$. Subsequently, player 1 receives a payoff $u(m, n)$ from player 2 . Then the game ends.

A special class of zero-sum games are binary games. Consider a set $W \subseteq A_{1} \times A_{2}$. The set $W$ is called the winning set of player 1 . In the binary game defined by $W$, the payoff function $u: A_{1} \times A_{2} \rightarrow\{0,1\}$ is the characteristic function of $W$. That is, $u(m, n)=1$ when $(m, n) \in W$, and $u(m, n)=0$ otherwise.

Example 3.1. The following binary game is a version of the Wald game (Wald, 1945). The action sets are $A_{1}=A_{2}=\mathbb{N}$. The payoff for $m \in A_{1}$ and $n \in A_{2}$ is $u(m, n)=1$ if $m \geq n$ and $u(m, n)=0$ if $m<n$. The payoffs are represented in the following matrix, where player 1 is the row player and player 2 is the column player.

\begin{tabular}{|c|cccc|}
\hline$u$ & 1 & 2 & 3 & $\ldots$ \\
\hline 1 & 1 & 0 & 0 & $\ldots$ \\
2 & 1 & 1 & 0 & $\ldots$ \\
3 & 1 & 1 & 1 & $\ldots$ \\
$\vdots$ & $\vdots$ & $\vdots$ & $\vdots$ & $\ddots$ \\
\hline
\end{tabular}

\subsection{Basic definitions}

Consider a zero-sum game $g(u)$ with payoff function $u$. A strategy for a player $i$ is a probability charge $\mu_{i} \in \mathcal{C}\left(A_{i}\right)$. A pair of strategies, one for each player, is called a strategy profile.

Consider a strategy profile $\left(\mu_{1}, \mu_{2}\right)$. In order to define the expected payoff for the players when they use this profile, we need a bit of notation.

A set $E \subseteq A_{1} \times A_{2}$ is called a rectangle if there are sets $E_{1} \subseteq A_{1}$ and $E_{2} \subseteq A_{2}$ with $E=E_{1} \times E_{2}$. A probability charge $\mu$ on $\mathcal{P}\left(A_{1} \times A_{2}\right)$ is called an extension of $\left(\mu_{1}, \mu_{2}\right)$ if for all rectangles $E=E_{1} \times E_{2}$ it holds that $\mu(E)=\mu_{1}\left(E_{1}\right) \cdot \mu_{2}\left(E_{2}\right)$. The set of extensions of $\left(\mu_{1}, \mu_{2}\right)$ is denoted by $<\mu_{1}, \mu_{2}>.^{6}$ All extensions of a given pair $\left(\mu_{1}, \mu_{2}\right)$ coincide on the algebra generated by the collection of rectangles. They may differ on other sets $E \subseteq A_{1} \times A_{2}$. Consequently, the expected payoff for the players will generally depend on the specific extension we consider. In this paper we closely study several specific ways to extend such a strategy profile $\left(\mu_{1}, \mu_{2}\right)$.

Formally, for a given extension $\mu$ of profile $\left(\mu_{1}, \mu_{2}\right)$, the expected payoff $U(\mu)$ is defined by

$$
U(\mu)=\int_{A_{1} \times A_{2}} u(m, n) d \mu .
$$

Thus, given a zero-sum game $g(u)$, this construction gives rise to many ways to define a game with charges. Let each player $i$ be endowed with a function $\tau_{i}: \mathcal{C}\left(A_{1}\right) \times \mathcal{C}\left(A_{2}\right) \rightarrow \mathcal{C}\left(A_{1} \times A_{2}\right)$. If for every profile $\left(\mu_{1}, \mu_{2}\right)$ we have that $\tau_{i}\left(\mu_{1}, \mu_{2}\right)$ is an extension of $\left(\mu_{1}, \mu_{2}\right)$, such a function is called an extension function. by

For given extension functions $\tau_{1}, \tau_{2}$, the expected payoffs $U^{\tau_{1}}\left(\mu_{1}, \mu_{2}\right)$ and $U^{\tau_{2}}\left(\mu_{1}, \mu_{2}\right)$ of profile $\left(\mu_{1}, \mu_{2}\right)$ are defined

$$
U^{\tau_{1}}\left(\mu_{1}, \mu_{2}\right)=U\left(\tau_{1}\left(\mu_{1}, \mu_{2}\right)\right) \text { and } U^{\tau_{2}}\left(\mu_{1}, \mu_{2}\right)=U\left(\tau_{2}\left(\mu_{1}, \mu_{2}\right)\right)
$$

\footnotetext{
4 The definition of a pure charge can be generalized to sets that are uncountably infinite. However, in the general case the definition is more involved.

5 In Theorem C.3 we can take for instance $\mathcal{F}$ to be the field of all finite subsets of $X$ and their complements.

6 Using Axiom of Choice, it can be shown that $\left\langle\mu_{1}, \mu_{2}>\right.$ is not empty. For more details, see Appendix C.
} 
Thus, in this paper we record payments for player 2 instead of rewards. This way we constructed a new two-player game $G\left(\tau_{1}, \tau_{2}\right)=\left(U^{\tau_{1}},-U^{\tau_{2}}\right)$. When $\tau_{1}=\tau_{2}$, the resulting game is again a zero-sum game. But in general this need not be the case.

Example 3.2. Let $W$ be the winning set of a binary game. Consider a strategy profile $\left(\mu_{1}, \mu_{2}\right)$ in this game. Then, for any extension function $\tau_{1}$, the payoff for player 1 is exactly

$$
U^{\tau_{1}}\left(\mu_{1}, \mu_{2}\right)=\int_{A_{1} \times A_{2}} u(m, n) d \tau_{1}\left(\mu_{1}, \mu_{2}\right)=\tau_{1}\left(\mu_{1}, \mu_{2}\right)(W) .
$$

The payment that player 2 expects to make is $U^{\tau_{2}}\left(\mu_{1}, \mu_{2}\right)=\tau_{2}\left(\mu_{1}, \mu_{2}\right)(W)$. Hence, his utility is $-U^{\tau_{2}}\left(\mu_{1}, \mu_{2}\right)$. $\triangleleft$

We will hardly ever explicitly specify the extension function in question, but simply explain the specific way extensions are constructed, and then leave it implicit how the game with charges is precisely defined.

\subsection{Optimistic and pessimistic players}

In this subsection we introduce two extreme cases of behavior, that depend on the attitude of the players. Our approach is based on the following Lemma.

Lemma 3.1. Let $\mu_{1} \in \mathcal{C}\left(A_{1}\right), \mu_{2} \in \mathcal{C}\left(A_{2}\right)$ and the bounded utility function $u$ be given. There exist extensions $\mu^{*}, \mu_{*} \in<\mu_{1}, \mu_{2}>$ with

$$
U\left(\mu^{*}\right) \geq U(\kappa) \text { and } U\left(\mu_{*}\right) \leq U(\kappa) \quad \text { for all } \kappa \in<\mu_{1}, \mu_{2}>.
$$

Proof. We construct $\mu^{*}$. The construction of $\mu_{*}$ is similar. For every $k \in \mathbb{N}$, let $v_{k}$ denote an extension of ( $\left.\mu_{1}, \mu_{2}\right)$ such that $U\left(v_{k}\right) \geq \sup _{\kappa \in<\mu_{1}, \mu_{2}>} U(\kappa)-\frac{1}{k}$. Take any pure probability charge $\tau$ on $\mathbb{N}$. Define the probability charge $\mu^{*}$ by

$$
\mu^{*}(E)=\int_{k \in \mathbb{N}} \nu_{k}(E) d \tau \quad \text { for every } E \in \mathcal{P}\left(A_{1} \times A_{2}\right) .
$$

Take a $K \in \mathbb{N}$ and $\kappa \in<\mu_{1}, \mu_{2}>$. We have

$$
U\left(\mu^{*}\right)=\int_{A_{1} \times A_{2}} u(m, n) d \mu^{*}=\int_{k \in \mathbb{N}} \int_{A_{1} \times A_{2}} u(m, n) d v_{k} d \tau=\int_{k \in \mathbb{N}} U\left(v_{k}\right) d \tau \geq U(\kappa)-\frac{1}{K},
$$

where the second equality comes from Lemma A.2, and the inequality is due to the fact that $\tau(\{K, K+1, \ldots\})=1$. Since $K$ was arbitrary, $U\left(\mu^{*}\right) \geq U(\kappa)$.

Player 1 is optimistic if, given profile $\left(\mu_{1}, \mu_{2}\right)$, he selects an extension $\mu^{*}$ with

$$
U\left(\mu^{*}\right) \geq U(\kappa) \text { for all } \kappa \in<\mu_{1}, \mu_{2}>.
$$

Due to the previous Lemma, such a selection is possible. The optimistic utility for player 1 is then defined by $U^{o}\left(\mu_{1}, \mu_{2}\right)=$ $U\left(\mu^{*}\right)$.

In the same way, player 1 is pessimistic, if he selects an extension $\mu_{*}$ with

$$
U\left(\mu_{*}\right) \leq U(\kappa) \text { for all } \kappa \in<\mu_{1}, \mu_{2}>\text {. }
$$

The pessimistic utility of player 1 is then defined by $U^{p}\left(\mu_{1}, \mu_{2}\right)=U\left(\mu_{*}\right)$.

Thus, an optimistic player 1 selects an extension $\mu^{*}$ of $\left(\mu_{1}, \mu_{2}\right)$ that, amongst all possible extensions, offers him the best expected payoff. A pessimistic player 1 selects an extension $\mu_{*}$ that offers him the worst expected payoff. However, since player 2 has to pay the amounts specified by the payoff function, an optimistic player 2 selects an extension $\mu_{*}$ that offers player 1 the worst expected payoff, and a pessimistic player 2 selects an extension $\mu^{*}$ of $\left(\mu_{1}, \mu_{2}\right)$ that, amongst all possible extensions, offers player 1 the best expected payoff. ${ }^{7}$

\footnotetext{
7 Given a profile $\left(\mu_{1}, \mu_{2}\right)$, the optimistic payoff of player 1 is equal to the infimum of the (uniquely defined) expected payoffs from step functions, as defined in Harris et al. (2005), that are larger than or equal to the payoff function for every profile of pure actions. This feature of optimistic expected payoff was pointed out to us by Ehud Lehrer. Of course a similar remark holds for pessimistic payoffs. Compare Lemma 1 of Lehrer (2009).
} 


\subsection{Double integrals}

As already mentioned in the Introduction, a stream of literature uses expected payoffs defined via double integrals as their starting point. This type of extension of a profile $\left(\mu_{1}, \mu_{2}\right)$ is defined as follows. For every set $E \in \mathcal{P}(\mathbb{N} \times \mathbb{N})$, define

$$
\mu(E)=\int_{m \in A_{1}} \int_{n \in A_{2}} \mathbb{I}_{E}(m, n) d \mu_{2} d \mu_{1}, 8
$$

where $\mathbb{I}_{E}$ denotes the characteristic function of the set $E$. We define $U^{21}\left(\mu_{1}, \mu_{2}\right)=U(\mu)$, where 21 refers to integrating first with respect to $\mu_{2}$ and then with respect to $\mu_{1}$. We have the following basic observation.

Lemma 3.2. It holds that

$$
U^{21}\left(\mu_{1}, \mu_{2}\right)=\int_{m \in A_{1}} \int_{n \in A_{2}} u(m, n) d \mu_{2} d \mu_{1}
$$

Proof. Let $\left(\mu_{1}, \mu_{2}\right)$ be a strategy profile. Let $\mu$ be the extension of $\left(\mu_{1}, \mu_{2}\right)$ as defined above. Take $X=A_{1} \times A_{2}$, and let $S$ be the set of all simple functions on $X$. Define for any bounded function $u$ on $X$

$$
\phi(u)=\int_{(m, n) \in X} u(m, n) d \mu .
$$

Define $\psi(u)$ by

$$
\psi(u)=\int_{m \in A_{1}} \int_{n \in A_{2}} u(m, n) d \mu_{2} d \mu_{1}
$$

It is straightforward to check that Lemma A.1 applies. This completes the proof.

We can also reverse the order of integration, the resulting expected utility function is denoted by $U^{12}\left(\mu_{1}, \mu_{2}\right)$.

Remark 3.1. Fix probability charges $\mu_{1} \in \mathcal{C}\left(A_{1}\right), \mu_{2} \in \mathcal{C}\left(A_{2}\right)$ and a bounded payoff function $u$. The Fubini-equality is

$$
\int_{m \in A_{1}} \int_{n \in A_{2}} u(m, n) d \mu_{2} d \mu_{1}=\int_{n \in A_{2}} \int_{m \in A_{1}} u(m, n) d \mu_{1} d \mu_{2}=\int_{A_{1} \times A_{2}} u(m, n) d \tau
$$

for any extension $\tau \in<\mu_{1}, \mu_{2}>$.

Fubini's theorem gives conditions under which the Fubini-equality holds. However, the following example shows that the Fubini-equality does not hold in general.

Example 3.3. Consider the Wald game again from Example 3.1. Take pure probability charges $\mu_{1} \in \mathcal{Q}\left(A_{1}\right)$ and $\mu_{2} \in \mathcal{Q}\left(A_{2}\right)$. In this case the Fubini-equality does not hold, since

$$
U^{21}\left(\mu_{1}, \mu_{2}\right)=\int_{m \in A_{1}} 0 d \mu_{1}=0 \quad \text { whereas } U^{12}\left(\mu_{1}, \mu_{2}\right)=\int_{n \in A_{2}} 1 d \mu_{2}=1 . \triangleleft
$$

\subsection{All games are different}

Thus, a single zero-sum game may generate different expected utility functions. In this section we show that different choices for extensions can result in different games with charges.

In general, of course, games induced by different extension functions might coincide, even though the extension functions are different. An extreme case are constant-payoff games, where all possible extension functions generate the same game. However, we have the following observation.

Proposition 3.3. Let $\left(\mu_{1}, \mu_{2}\right)$ be a strategy profile. Let $\kappa$ and $\mu$ be two different extensions of this profile. Then there is a zero-sum game $g(u)$ with $U(\kappa) \neq U(\mu)$.

\footnotetext{
8 In this case we first integrate the function $\mathbb{I}_{E}(m, n)$ with respect to $\mu_{2}$. This integral will depend only on $m$. Then we integrate with respect to $\mu_{1}$.
} 
Proof. Since $\kappa \neq \mu$, there is a set $W$ with $\kappa(W) \neq \mu(W)$. Let $g(u)$ be the binary game with winning set $W$. Then $U(\kappa)=$ $\kappa(W) \neq \mu(W)=U(\mu)$.

For the specific cases of $U^{0}, U^{p}, U^{21}$ and $U^{12}$, we present a zero-sum game $g(u)$ for which all four extensions are different. More precisely, we present a binary game $g(u)$ and strategies $\mu_{1} \in \mathcal{C}(\mathbb{N}), \mu_{2} \in \mathcal{C}(\mathbb{N})$, and $\kappa_{1} \in \mathcal{C}(\mathbb{N})$ with $U^{p}\left(\mu_{1}, \mu_{2}\right)=0, U^{o}\left(\mu_{1}, \mu_{2}\right)=1, U^{21}\left(\mu_{1}, \mu_{2}\right)=U^{12}\left(\mu_{1}, \mu_{2}\right)=\frac{1}{2}, U^{21}\left(\kappa_{1}, \mu_{2}\right)=1$ and $U^{12}\left(\kappa_{1}, \mu_{2}\right)=0$. Thus, $U^{o}, U^{p}, U^{21}$ and $U^{12}$ define four different games, even though they are derived from the same zero-sum game.

Example 3.4. Consider the following binary game. Let $A_{1}=A_{2}=\mathbb{N}$. When player 1 plays $m$ and player 2 plays $n$, the payoff $u(m, n)$ is defined by

$$
u(m, n)= \begin{cases}1 & \text { if } m \geq n \text { and } m \text { is odd } \\ 1 & \text { if } m \leq n \text { and } m \text { is even } \\ 0 & \text { otherwise. }\end{cases}
$$

The game can be represented as follows.

\begin{tabular}{|l|lllllll|}
\hline$u$ & 1 & 2 & 3 & 4 & 5 & 6 & $\ldots$ \\
\hline 1 & 1 & 0 & 0 & 0 & 0 & 0 & $\ldots$ \\
2 & 0 & 1 & 1 & 1 & 1 & 1 & $\ldots$ \\
3 & 1 & 1 & 1 & 0 & 0 & 0 & $\ldots$ \\
4 & 0 & 0 & 0 & 1 & 1 & 1 & $\ldots$ \\
5 & 1 & 1 & 1 & 1 & 1 & 0 & $\ldots$ \\
6 & 0 & 0 & 0 & 0 & 0 & 1 & $\ldots$ \\
$\vdots$ & $\vdots$ & $\vdots$ & $\vdots$ & $\vdots$ & $\vdots$ & $\vdots$ & $\ddots$ \\
\hline
\end{tabular}

Write $E=\{m \in \mathbb{N}: m$ is even $\}$ and $O=\{m \in \mathbb{N}: m$ is odd $\}$. Take a pure probability charge $\mu_{1}$ for player 1 such that

$$
\mu_{1}(E)=\mu_{1}(O)=\frac{1}{2} .9
$$

Let $\mu_{2}$ be an arbitrary pure probability charge for player 2 . Then for the double integrals we have

$$
\begin{aligned}
U^{12}\left(\mu_{1}, \mu_{2}\right) & =\int_{n \in \mathbb{N}} \int_{m \in O} u(m, n) d \mu_{1} d \mu_{2}+\int_{n \in \mathbb{N}} \int_{m \in E} u(m, n) d \mu_{1} d \mu_{2} \\
& =\int_{n \in \mathbb{N}} \int_{\substack{m \in O \\
m \geq n}} 1 d \mu_{1} d \mu_{2}+\int_{\substack{n \in \mathbb{N} \\
m_{m \in E}}} 0 d \mu_{1} d \mu_{2} \\
& =\int_{n \in \mathbb{N}} \frac{1}{2} d \mu_{2}+\int_{n \in \mathbb{N}} 0 d \mu_{2}=\frac{1}{2}+0=\frac{1}{2} .
\end{aligned}
$$

Similarly,

$$
\begin{aligned}
U^{21}\left(\mu_{1}, \mu_{2}\right) & =\int_{m \in O} \int_{n \in \mathbb{N}} u(m, n) d \mu_{2} d \mu_{1}+\int_{m \in E} \int_{n \in \mathbb{N}} u(m, n) d \mu_{2} d \mu_{1} \\
& =\int_{m \in O} 0 d \mu_{1}+\int_{m \in E} 1 d \mu_{1}=0+\frac{1}{2}=\frac{1}{2}
\end{aligned}
$$

We verify that $U^{p}\left(\mu_{1}, \mu_{2}\right)=0$. Write

$$
K=\{(m, n) \in \mathbb{N} \times \mathbb{N} \mid m \text { is even and } m \leq n\} .
$$

Define, as in Appendix C,

$$
\alpha\left(\mu_{1}, \mu_{2}\right)(K)=\sup \left\{\sum_{i=1}^{k} \mu_{1}\left(C_{i}\right) \cdot \mu_{2}\left(D_{i}\right) \mid C_{i} \times D_{i} \text { are mutually disjoint, contained in } K\right\} .
$$

\footnotetext{
${ }^{9}$ By Theorem C.3, we can in fact construct such a pure charge.
} 
We show that $\alpha\left(\mu_{1}, \mu_{2}\right)(K)=0$. Let $A \times B$ be a non-empty rectangle with $A \times B \subseteq K$. We show that $\mu_{1}(A) \cdot \mu_{2}(B)=0$. Suppose that $A$ is infinite. Take any $n \in B$. Since $A$ is infinite, there is $m \in A$ with $m>n$. Then $m>n$ and $(m, n) \in K$, which contradicts the definition of $K$. So, $A$ is finite. Hence, since $\mu_{1}$ is a pure charge, $\mu_{1}(A) \cdot \mu_{2}(B)=0$. It follows that $\alpha\left(\mu_{1}, \mu_{2}\right)(K)=0$.

Now define

$$
L=\{(m, n) \in \mathbb{N} \times \mathbb{N} \mid m \text { is odd and } m \geq n\} .
$$

Since also $\mu_{2}$ is a pure charge, we can show that $\alpha\left(\mu_{1}, \mu_{2}\right)(L)=0$. However, let $W$ denote the winning set of the game. Then $W=K \cup L$, so that also $\alpha\left(\mu_{1}, \mu_{2}\right)(W)=0$. The claim that $U^{p}\left(\mu_{1}, \mu_{2}\right)=0$ then follows from Proposition C.5. In the same way we can verify that $U^{o}\left(\mu_{1}, \mu_{2}\right)=1$.

Now, let $\kappa_{1}$ be a pure charge with $\kappa_{1}(E)=1$. Again, such a charge exists by Theorem C.3. Then $U^{21}\left(\kappa_{1}, \mu_{2}\right)=1$ and $U^{12}\left(\kappa_{1}, \mu_{2}\right)=0$.

Remark 3.2. The above example distinguishes our work from the work by Schervish and Seidenfeld (1996), and Capraro and Scarsini (2013). Those two articles consider convex combinations of the different orders of integration. Note that the optimistic and pessimistic approaches are not of that type. In the above example, $U^{21}\left(\mu_{1}, \mu_{2}\right)=U^{12}\left(\mu_{1}, \mu_{2}\right)=\frac{1}{2}$. So, convex combinations of these two payoff functions also result in a payoff of $\frac{1}{2}$. But the pessimistic and optimistic expected payoffs are different in this case, namely $U^{p}\left(\mu_{1}, \mu_{2}\right)=0$ and $U^{o}\left(\mu_{1}, \mu_{2}\right)=1$.

\section{Guarantee level}

Thus, a single zero-sum game $g(u)$ generates many different games with charges. We study the solutions for a few of the more natural and interesting choices out of the various possibilities.

\subsection{Definitions}

Let $g(u)$ be a zero-sum game. When $\left(\mu_{1}, \mu_{2}\right)$ has a unique extension $\mu$, we write $U\left(<\mu_{1}, \mu_{2}>\right)$ instead of $U(\mu)$. Now we turn to the definition of a guarantee level in probability charges. We first briefly discuss the definition for general extension functions. Let $g(u)$ be a zero-sum game, and let $\tau_{1}: \mathcal{C}\left(A_{1}\right) \times \mathcal{C}\left(A_{2}\right) \rightarrow \mathcal{C}\left(A_{1} \times A_{2}\right)$ and $\tau_{2}: \mathcal{C}\left(A_{1}\right) \times \mathcal{C}\left(A_{2}\right) \rightarrow$ $\mathcal{C}\left(A_{1} \times A_{2}\right)$ be two extension functions. The resulting game is $\left(U^{\tau_{1}},-U^{\tau_{2}}\right) .{ }^{10}$

Definition 4.1. The $\tau_{1}$-guarantee level $v_{1}^{\tau_{1}}$ for player 1 , and the $\tau_{2}$-guarantee level $v_{2}^{\tau_{2}}$ for player 2 are defined by

$$
v_{1}^{\tau_{1}}=\sup _{\mu_{1} \in \mathcal{C}\left(A_{1}\right)} \inf _{\mu_{2} \in \mathcal{C}\left(A_{2}\right)} U^{\tau_{1}}\left(\mu_{1}, \mu_{2}\right) \quad \text { and } v_{2}^{\tau_{2}}=\inf _{\mu_{2} \in \mathcal{C}\left(A_{2}\right)} \sup _{\mu_{1} \in \mathcal{C}\left(A_{1}\right)} U^{\tau_{2}}\left(\mu_{1}, \mu_{2}\right) .
$$

If the two extension functions coincide, that is $\tau=\tau_{1}=\tau_{2}$, and $v_{1}^{\tau}=v_{2}^{\tau}$, then we say that the $\tau$-value $v^{\tau}$ of the resulting game $\left(U^{\tau},-U^{\tau}\right)$ exists.

In the specific cases that we mainly study-optimistic and pessimistic extensions, and double integrals-the definitions look as follows.

Definition 4.2. The optimistic guarantee level $v_{1}^{o}$ for player 1 , and the optimistic guarantee level $v_{2}^{o}$ for player 2 are defined by

$$
v_{1}^{o}=\sup _{\mu_{1} \in \mathcal{C}\left(A_{1}\right)} \inf _{\mu_{2} \in \mathcal{C}\left(A_{2}\right)} U^{o}\left(\mu_{1}, \mu_{2}\right) \quad \text { and } \quad v_{2}^{o}=\inf _{\mu_{2} \in \mathcal{C}\left(A_{2}\right)} \sup _{\mu_{1} \in \mathcal{C}\left(A_{1}\right)} U^{p}\left(\mu_{1}, \mu_{2}\right)
$$

The pessimistic guarantee level $v_{1}^{p}$ for player 1 , and the pessimistic guarantee level $v_{2}^{p}$ for player 2 are defined by

$$
v_{1}^{p}=\sup _{\mu_{1} \in \mathcal{C}\left(A_{1}\right)} \inf _{\mu_{2} \in \mathcal{C}\left(A_{2}\right)} U^{p}\left(\mu_{1}, \mu_{2}\right) \quad \text { and } \quad v_{2}^{p}=\inf _{\mu_{2} \in \mathcal{C}\left(A_{2}\right)} \sup _{\mu_{1} \in \mathcal{C}\left(A_{1}\right)} U^{o}\left(\mu_{1}, \mu_{2}\right) .
$$

The 21-guarantee level $v_{1}^{21}$ for player 1 , and the 21 -guarantee level $v_{2}^{21}$ for player 2 are defined by

$$
v_{1}^{21}=\sup _{\mu_{1} \in \mathcal{C}\left(A_{1}\right)} \inf _{\mu_{2} \in \mathcal{C}\left(A_{2}\right)} U^{21}\left(\mu_{1}, \mu_{2}\right) \quad \text { and } v_{2}^{21}=\inf _{\mu_{2} \in \mathcal{C}\left(A_{2}\right)} \sup _{\mu_{1} \in \mathcal{C}\left(A_{1}\right)} U^{21}\left(\mu_{1}, \mu_{2}\right) .
$$

In the same way we can define $v_{1}^{12}$ and $v_{2}^{12}$. The fact that the pessimistic guarantee level for player 2 involves $U^{0}$ and the optimistic guarantee level involves $U^{p}$ is due to the fact that player 2 still views the amounts specified by the utility function as payments, not as rewards.

\footnotetext{
10 Thus, given a strategy profile $\left(\mu_{1}, \mu_{2}\right)$, even though the players do not need to have an agreement on the outcome of the game, we still assume that player 1 expects to receive an amount $U^{\tau_{1}}\left(\mu_{1}, \mu_{2}\right)$, while player 2 expects to pay the amount $U^{\tau_{2}}\left(\mu_{1}, \mu_{2}\right)$.
} 


\subsection{Finitistic guarantee levels}

We also introduce several guarantee levels that do not originate from extensions of strategy profiles, but from approximations of a (zero-sum) game by finite games. Let $\mathcal{D}\left(A_{i}\right)$ be the set of probability charges (or equivalently, measures) for player $i$ with finite support. Note that $\mathcal{D}\left(A_{i}\right)$ is dense in $\mathcal{C}\left(A_{i}\right)$ with respect to the topology of weak convergence. ${ }^{11}$ We define

$$
v_{1}^{d}=\sup _{\mu_{1} \in \mathcal{D}\left(A_{1}\right)} \inf _{\mu_{2} \in \mathcal{D}\left(A_{2}\right)} U\left(<\mu_{1}, \mu_{2}>\right) \quad \text { and } \quad v_{2}^{d}=\inf _{\mu_{2} \in \mathcal{D}\left(A_{2}\right)} \sup _{\mu_{1} \in \mathcal{D}\left(A_{1}\right)} U\left(<\mu_{1}, \mu_{2}>\right) .
$$

For two non-empty finite sets $F_{1} \in \mathcal{F}\left(A_{1}\right)$ and $F_{2} \in \mathcal{F}\left(A_{2}\right)$, consider the game $G\left(F_{1}, F_{2}\right)$ in which player 1 is restricted to $F_{1}$ and player 2 is restricted to $F_{2}$. This game has a value, say $v\left(F_{1}, F_{2}\right)$. We define

$$
v_{1}^{f}=\sup _{F_{1} \in \mathcal{F}\left(A_{1}\right)} \inf _{F_{2} \in \mathcal{F}\left(A_{2}\right)} v\left(F_{1}, F_{2}\right) \quad \text { and } \quad v_{2}^{f}=\inf _{F_{2} \in \mathcal{F}\left(A_{2}\right)} \sup _{F_{1} \in \mathcal{F}\left(A_{1}\right)} v\left(F_{1}, F_{2}\right) \text {. }
$$

\subsection{The main result}

We provide the precise relationship between all guarantee levels defined above in the following Theorem. The result is surprising in the sense that all guarantee levels appear to coincide, despite the fact that the games from which they are derived are, in general, all different. The proof of Theorem 4.1 can be found in Appendix B, Theorem B.2.

Theorem 4.1. Let $g(u)$ be any zero-sum game. The guarantee levels of the players satisfy

$$
v_{1}^{f}=v_{1}^{d}=v_{1}^{p}=v_{2}^{o}=v_{1}^{21}=v_{2}^{21} \quad \text { and } \quad v_{2}^{f}=v_{2}^{d}=v_{2}^{p}=v_{1}^{o}=v_{2}^{12}=v_{1}^{12} \text {. }
$$

Note that, even though the double integral $U^{21}$ might differ from the pessimistic expected payoff $U^{p}$, the corresponding guarantee levels for player 1 coincide. That is, $v_{1}^{21}=v_{1}^{p}$.

We identify a specific class of games for which all guarantee levels in Theorem 4.1 coincide. We say that a function is a simple function when it is a finite linear combination of indicator functions of sets of the form $A \times B$, with $A \subseteq A_{1}$ and $B \subseteq A_{2}$. Harris et al. (2005) call a payoff function $u: A_{1} \times A_{2} \rightarrow \mathbb{R}$ integrable when $u$ is the uniform limit of simple functions. An integrable payoff function's expected payoff is uniquely defined for any strategy profile. Marinacci (1997) and Harris et al. (2005) prove the existence of Nash-equilibrium in nonzero-sum games with integrable payoff functions.

We have the following result, which extends the classic result of von Neumann (1928).

Corollary 4.2. Let $g(u)$ be a zero-sum game, where $u$ is an integrable function. Then all guarantee levels are equal.

Proof. For an integrable function, the Fubini-equality (cf. Remark 3.1) holds for any pair of probability charges. For a detailed proof, see Marinacci (1997, Proposition 3). This implies that $v_{1}^{21}=v_{1}^{12}$. The result is now a direct consequence of Theorem 4.1.

This result is also in line with the findings of Harris et al. (2005). They show that, under the condition that the utility functions of an $n$-person normal form game are integrable, the resulting game played with charges is uniquely defined, and admits a Nash equilibrium.

\subsection{The classical guarantee level}

In this subsection we examine the classical guarantee levels of the players, which are defined through countably additive strategies. We restrict our attention to games where the action spaces of the players are countable. First we provide a formal definition of these classical guarantee levels, and then in Theorem 4.3 we relate them to the guarantee levels defined through charges and to the finitistic guarantee levels. As we show, the classical guarantee level of each player coincides with his pessimistic guarantee level.

Suppose that $A_{1}=A_{2}=\mathbb{N}$. A zero-sum game $g(u)$ defined by a bounded payoff function $u: \mathbb{N} \times \mathbb{N} \rightarrow \mathbb{R}$ is called countably infinite. ${ }^{12}$ For this case, the (classical) guarantee level for player 1 and, respectively, the (classical) guarantee level for player 2 are defined as

$$
v_{1}=\sup _{p_{1} \in \Delta\left(A_{1}\right)} \inf _{p_{2} \in \Delta\left(A_{2}\right)} U\left(<p_{1}, p_{2}>\right) \quad \text { and } \quad v_{2}=\inf _{p_{2} \in \Delta\left(A_{2}\right)} \sup _{p_{1} \in \Delta\left(A_{1}\right)} U\left(<p_{1}, p_{2}>\right) .
$$

\footnotetext{
11 Note that this topology is not metrizable, so one needs to work with nets and not only sequences.

12 Note that the choice of $\mathbb{N}$ is not relevant, only the cardinality matters. The results hold for any set with countable cardinality.
} 
Theorem 4.3. Let $g(u)$ be any countably infinite zero-sum game. The guarantee levels of the players satisfy

$$
v_{1}=v_{1}^{f}=v_{1}^{d}=v_{1}^{p}=v_{2}^{o}=v_{1}^{21}=v_{2}^{21} \quad \text { and } \quad v_{2}=v_{2}^{f}=v_{2}^{d}=v_{2}^{p}=v_{1}^{o}=v_{2}^{12}=v_{1}^{12} .
$$

Proof. A. We show that $v_{1}^{d} \leq v_{1}$. Take $\mu_{1} \in \mathcal{D}(\mathbb{N})$. Then

$$
\inf _{\mu_{2} \in \Delta(\mathbb{N})} U\left(<\mu_{1}, \mu_{2}>\right)=\inf _{n \in \mathbb{N}} U\left(<\mu_{1}, \delta(n)>\right)=\inf _{\mu_{2} \in \mathcal{D}(\mathbb{N})} U\left(<\mu_{1}, \mu_{2}>\right) .
$$

Therefore,

$$
\begin{aligned}
v_{1} & =\sup _{\mu_{1} \in \Delta(\mathbb{N})} \inf _{\mu_{2} \in \Delta(\mathbb{N})} U\left(<\mu_{1}, \mu_{2}>\right) \\
& \geq \sup _{\mu_{1} \in \mathcal{D}(\mathbb{N})} \inf _{\mu_{2} \in \Delta(\mathbb{N})} U\left(<\mu_{1}, \mu_{2}>\right) \\
& =\sup _{\mu_{1} \in \mathcal{D}(\mathbb{N})} \inf _{\mu_{2} \in \mathcal{D}(\mathbb{N})} U\left(<\mu_{1}, \mu_{2}>\right) \\
& =v_{1}^{d} .
\end{aligned}
$$

B. We show that $v_{1} \leq v_{1}^{21}$. Take any $p_{1} \in \Delta(\mathbb{N})$ and any $\mu_{2} \in \mathcal{C}(\mathbb{N})$. Then by Lemma A.3

$$
\begin{aligned}
U^{21}\left(p_{1}, \mu_{2}\right)=U\left(<p_{1}, \mu_{2}>\right) & =\int_{n \in \mathbb{N}} \int_{m \in \mathbb{N}} u(m, n) d p_{1} d \mu_{2} \\
& \geq \int_{n \in \mathbb{N}}\left(\inf _{n \in \mathbb{N}} U\left(<p_{1}, \delta(n)>\right)\right) d \mu_{2} \\
& =\inf _{n \in \mathbb{N}} U\left(<p_{1}, \delta(n)>\right) \\
& =\inf _{p_{2} \in \Delta(\mathbb{N})} U\left(<p_{1}, p_{2}>\right) .
\end{aligned}
$$

So, since the last expression does not depend on $\mu_{2}$,

$$
\inf _{\mu_{2} \in \mathcal{C}(\mathbb{N})} U^{21}\left(p_{1}, \mu_{2}\right) \geq \inf _{p_{2} \in \Delta(\mathbb{N})} U\left(<p_{1}, p_{2}>\right) .
$$

Hence,

$$
\begin{aligned}
v_{1}^{21} & =\sup _{\mu_{1} \in \mathcal{C}(\mathbb{N})} \inf _{\mu_{2} \in \mathcal{C}(\mathbb{N})} U^{21}\left(\mu_{1}, \mu_{2}\right) \\
& \geq \sup _{p_{1} \in \Delta(\mathbb{N})} \inf _{\mu_{2} \in \mathcal{C}(\mathbb{N})} U^{21}\left(p_{1}, \mu_{2}\right) \\
& \geq \sup _{p_{1} \in \Delta(\mathbb{N})} \inf _{p_{2} \in \Delta(\mathbb{N})} U\left(<p_{1}, p_{2}>\right) \\
& =v_{1} .
\end{aligned}
$$

The first half of the assertion now follows from Theorem 4.1. The proof of the second half is similar.

We always have $v_{1} \leq v_{2}$. Theorem 4.3 has the following corollary for the case when $v_{1}=v_{2}$ holds, that is, when the classical value exists.

Corollary 4.4. The classical value exists in a countably infinite zero-sum game if and only if all guarantee levels in Theorem 4.3 coincide.

\section{Optimality}

When using countably additive strategies, even though the value of a game may exist, players may have only approximate optimal strategies. We investigate to which extent the use of charges guarantees existence of optimal strategies.

Let $g(u)$ be a zero-sum game, and let $\tau_{1}: \mathcal{C}\left(A_{1}\right) \times \mathcal{C}\left(A_{2}\right) \rightarrow \mathcal{C}\left(A_{1} \times A_{2}\right)$ and $\tau_{2}: \mathcal{C}\left(A_{1}\right) \times \mathcal{C}\left(A_{2}\right) \rightarrow \mathcal{C}\left(A_{1} \times A_{2}\right)$ be the extension functions employed by the respective players. Thus the resulting game is $\left(U^{\tau_{1}},-U^{\tau_{2}}\right)$.

Definition 5.1. A probability charge $\mu_{1} \in \mathcal{C}\left(A_{1}\right)$ is a $\tau_{1}$-optimal strategy for player 1 if for all strategies $\mu_{2} \in \mathcal{C}\left(A_{2}\right)$ it holds that

$$
U^{\tau_{1}}\left(\mu_{1}, \mu_{2}\right) \geq v_{1}^{\tau_{1}}
$$


A probability charge $\mu_{2} \in \mathcal{C}\left(A_{2}\right)$ is a $\tau_{2}$-optimal strategy for player 2 if for all strategies $\mu_{1} \in \mathcal{C}\left(A_{1}\right)$ it holds that

$$
U^{\tau_{2}}\left(\mu_{1}, \mu_{2}\right) \leq v_{2}^{\tau_{2}}
$$

\subsection{Optimistic optimality}

When player 1 uses $U^{o}$ to evaluate outcomes, we say that an optimal strategy for player 1 is optimistic optimal. When player 2 uses $-U^{p}$ to evaluate outcomes, we say that an optimal strategy for player 2 is optimistic optimal. We have the following result.

Theorem 5.1. Both players have optimistic optimal strategies.

Proof. By Lemma B.1, player 2 has a strategy $\sigma_{2} \in \mathcal{C}\left(A_{2}\right)$ such that for every $\mu_{1} \in \mathcal{C}\left(A_{1}\right)$ it holds that

$$
U^{21}\left(\mu_{1}, \sigma_{2}\right) \leq v_{1}^{f}
$$

Since $v_{1}^{f}=v_{2}^{o}$ by Theorem 4.1, it follows that $\sigma_{2}$ is optimistic optimal for player 2. The proof for player 1 is similar.

Remark 5.1. We show how to construct optimistic optimal strategies directly. For each $k \in \mathbb{N}$, let $p_{1 k} \in \Delta\left(A_{1}\right)$ be such that, for every $p_{2} \in \Delta\left(A_{2}\right)$,

$$
U\left(\left\langle p_{1 k}, p_{2}\right\rangle\right) \geq v-\frac{1}{k}
$$

Let $\mu_{1}$ be a pure charge. Define the probability charge $\kappa_{1}$ by

$$
\kappa_{1}(E)=\int_{k \in \mathbb{N}} p_{1 k}(E) d \mu_{1} \quad \text { for all } E \subseteq A_{1} \times A_{2} .
$$

Let $\mu_{2}$ be any probability charge of player 2 . Take a $K \in \mathbb{N}$. Then for every $n \in A_{2}$,

$$
\begin{aligned}
\int_{m \in A_{1}} u(m, n) d \kappa_{1} & =\int_{k \in \mathbb{N}} \int_{m \in A_{1}} u(m, n) d p_{1 k} d \mu_{1} \\
& =\int_{k \geq K} \int_{m \in A_{1}} u(m, n) d p_{1 k} d \mu_{1} \\
& \geq \int_{k \geq K}\left(v-\frac{1}{k}\right) d \mu_{1} \geq v-\frac{1}{K} .
\end{aligned}
$$

The first equality comes from Lemma A.2, and the second equality is due to the fact that $\mu_{1}(\{K, K+1, \ldots\})=1$. Since $K$ was chosen arbitrarily, we find that

$$
\int_{m \in A_{1}} u(m, n) d \kappa_{1} \geq v
$$

Then for every probability charge $\mu_{2}$ of player 2 ,

$$
U^{o}\left(\kappa_{1}, \mu_{2}\right) \geq \int_{n \in A_{2}} \int_{m \in A_{1}} u(m, n) d \kappa_{1} d \mu_{2} \geq \int_{n \in A_{2}} v d \mu_{2}=v .
$$

Hence, $\kappa_{1}$ is optimistic optimal for player $1 . \triangleleft$

Example 5.1. Also in zero-sum games that do not have a classical value, players have optimistic optimal strategies. Consider the Wald game

\begin{tabular}{|c|cccc|}
\hline$u$ & 1 & 2 & 3 & $\ldots$ \\
\hline 1 & 1 & 0 & 0 & $\ldots$ \\
2 & 1 & 1 & 0 & $\ldots$ \\
3 & 1 & 1 & 1 & $\ldots$ \\
$\vdots$ & $\vdots$ & $\vdots$ & $\vdots$ & $\ddots$ \\
\hline
\end{tabular}


defined in Example 3.1. It is well-known that this game does not have a classical value, and countably additive optimal strategies do not exist.

We show that $v_{1}^{o}=1$ and that the set of optimistic optimal strategies for player 1 is exactly the set $\mathcal{Q}(\mathbb{N})$ of pure probability charges. Take $\kappa_{1} \in \mathcal{Q}(\mathbb{N})$ and take any $\mu_{2} \in \mathcal{C}(\mathbb{N})$. We verify that $U^{o}\left(\kappa_{1}, \mu_{2}\right)=1$. Write

$$
W=\{(m, n) \in \mathbb{N} \times \mathbb{N} \mid m \geq n\} .
$$

We show that $\beta\left(\kappa_{1}, \mu_{2}\right)(K)=1$, where

$$
\beta\left(\kappa_{1}, \mu_{2}\right)(K)=\inf \left\{\sum_{i=1}^{k} \kappa_{1}\left(C_{i}\right) \cdot \mu_{2}\left(D_{i}\right) \mid C_{i} \times D_{i} \text { are mutually disjoint, and cover } K\right\}
$$

as defined in Appendix C. Let $A \times B$ be a non-empty rectangle with $A \times B \cap W=\phi$. We show that $\kappa_{1}(A) \cdot \mu_{2}(B)=0$. Suppose that $A$ is infinite. Take any $n \in B$. Since $A$ is infinite, there is $m \in A$ with $m \geq n$. Then $(m, n) \in W$, which contradicts the assumption that $A \times B \cap W=\phi$. So, $A$ is finite. Hence, since $\mu_{1}$ is a pure charge, $\mu_{1}(A) \cdot \mu_{2}(B)=0$. It follows that $\beta\left(\kappa_{1}, \mu_{2}\right)(W)=1$.

So, by Proposition C.5 there exists an extension $\mu$ of $\left(\kappa_{1}, \mu_{2}\right)$ such that $\mu(W)=1$. This implies that $U^{o}\left(\kappa_{1}, \mu_{2}\right)=1$. Since $\mu_{2} \in \mathcal{C}(\mathbb{N})$ was chosen arbitrarily, it follows that $v_{1}^{o}=1$, and $\kappa_{1}$ is optimistic optimal for player 1 .

Now take $\mu_{1} \in \mathcal{C}(\mathbb{N})$ with $\mu_{1} \notin \mathcal{Q}(\mathbb{N})$. We show that $\mu_{1}$ is not optimistic optimal for player 1 . Let $\kappa_{2} \in \mathcal{Q}(\mathbb{N})$. Write $\mu_{1}=\lambda \cdot p_{1}+(1-\lambda) \cdot \kappa_{1}$ with $p_{1} \in \Delta(\mathbb{N}), \kappa_{1} \in \mathcal{Q}(\mathbb{N})$ and $\lambda>0$. Then

$$
U^{o}\left(\mu_{1}, \kappa_{2}\right)=\lambda \cdot U^{o}\left(p_{1}, \kappa_{2}\right)+(1-\lambda) \cdot U^{o}\left(\kappa_{1}, \kappa_{2}\right)=(1-\lambda) \cdot U^{o}\left(\kappa_{1}, \kappa_{2}\right)<1 .
$$

Hence, $\mu_{1}$ is not optimistic optimal for player $1 . \triangleleft$

Example 5.2. We show that an optimistic optimal strategy may be neither countably additive nor a pure probability charge, and may have to be a real mixture of the two.

Again consider the game from Example 3.4

\begin{tabular}{|c|ccccccc|}
\hline$u$ & 1 & 2 & 3 & 4 & 5 & 6 & $\ldots$ \\
\hline 1 & 1 & 0 & 0 & 0 & 0 & 0 & $\ldots$ \\
2 & 0 & 1 & 1 & 1 & 1 & 1 & $\ldots$ \\
3 & 1 & 1 & 1 & 0 & 0 & 0 & $\ldots$ \\
4 & 0 & 0 & 0 & 1 & 1 & 1 & $\ldots$ \\
5 & 1 & 1 & 1 & 1 & 1 & 0 & $\ldots$ \\
6 & 0 & 0 & 0 & 0 & 0 & 1 & $\ldots$ \\
$\vdots$ & $\vdots$ & $\vdots$ & $\vdots$ & $\vdots$ & $\vdots$ & $\vdots$ & $\ddots$ \\
\hline
\end{tabular}

First we show that $v_{2}^{o}=\frac{1}{2}$. Suppose that player 1 plays the first row with probability $\frac{1}{2}$ and the second row with probability $\frac{1}{2}$. Then the expected payoff is exactly $\frac{1}{2}$ regardless of what player 2 does. So, $v_{1} \geq \frac{1}{2}$.

Conversely, let $p \in \Delta(\mathbb{N})$ be any countably additive strategy for player 1 . Let $\varepsilon>0$. Take a number $m$ such that $\sum_{k=m}^{\infty} p(k)<\varepsilon$. Take $n$ with $n>m$, and let player 2 play the first column with probability $\frac{1}{2}$ and column $n$ with probability $\frac{1}{2}$. Then the expected payoff for player 1 is at most $\frac{1}{2}+\varepsilon$. These observations imply that $v_{1}=\frac{1}{2}$. Hence, by Theorem 4.3 also $v_{2}^{o}=\frac{1}{2}$.

Now notice that for any pure probability charge $\mu_{1}$ with $\mu_{1}(0)=1$ (where 0 stands for the set of odd numbers) and for any countably additive strategy $p_{2}$, we have $U\left(<\mu_{1}, p_{2}>\right)=1$. Hence, countably additive strategies are not optimistic optimal for player 2 .

Next, if player 1 uses the Dirac measure $\delta(2)$ on action 2, and player 2 uses a pure probability charge $\mu_{2}$, then $U\left(<\delta(2), \mu_{2}>\right)=1$. Hence, an optimistic optimal strategy for player 2 cannot be a pure probability charge either.

Consequently, any optimistic optimal strategy for player 2 must be a real mixture of a countably additive strategy and a pure probability charge. For completeness, we provide an optimistic optimal strategy for player 2 . Let $\mu_{2}=\frac{1}{2} \cdot \delta(1)+\frac{1}{2} \cdot \kappa_{2}$ where $\delta(1)$ is the Dirac measure on action 1 and $\kappa$ is any pure probability charge on $\mathbb{N}$. Take a strategy $\mu_{1}$ of player 1 . We write $d_{1}=\sum_{m \in \mathbb{N}} \mu_{1}(m)$, and $\mu_{1}=d_{1} p_{1}+\left(1-d_{1}\right) \kappa_{1}$ with $p_{1} \in \Delta(\mathbb{N})$ and $\kappa_{1} \in \mathcal{Q}(\mathbb{N})$. Then

$$
\begin{aligned}
U^{p}\left(\mu_{1}, \mu_{2}\right)= & \frac{1}{2} \cdot d_{1} \cdot U\left(<p_{1}, \delta(1)>\right)+\frac{1}{2} \cdot\left(1-d_{1}\right) \cdot U\left(<\kappa_{1}, \delta(1)>\right) \\
& +\frac{1}{2} \cdot d_{1} \cdot U\left(<p_{1}, \kappa_{2}>\right)+\frac{1}{2} \cdot\left(1-d_{1}\right) U^{p}\left(\kappa_{1}, \kappa_{2}\right) \\
= & \frac{1}{2} \cdot d_{1} \cdot p_{1}(O)+\frac{1}{2} \cdot\left(1-d_{1}\right) \cdot \kappa_{1}(O)+\frac{1}{2} \cdot d_{1} \cdot p_{1}(E)+\frac{1}{2} \cdot\left(1-d_{1}\right) \cdot 0 \\
= & \frac{1}{2} \cdot d_{1}+\frac{1}{2} \cdot\left(1-d_{1}\right) \cdot \kappa_{1}(O) \\
\leq & \frac{1}{2} \cdot d_{1}+\frac{1}{2} \cdot\left(1-d_{1}\right) \\
= & \frac{1}{2} .
\end{aligned}
$$


Here, the inequality follows from $\kappa_{1}(0) \leq 1$. Since $v_{2}^{o}=\frac{1}{2}$, the strategy $\mu_{2}$ is optimistic optimal for player 2 . $\triangleleft$

\subsection{Pessimistic optimality}

When player 1 uses $U^{p}$ to evaluate outcomes, we say that an optimal strategy for player 1 is pessimistic optimal. When player 2 uses $-U^{o}$ to evaluate outcomes, we say that an optimal strategy for player 2 is pessimistic optimal.

Example 5.3. We do not know whether pessimistic optimal strategies always exist. The game below shows that the set of pessimistic optimal strategies may be non-trivial. We show that in this example pessimistic optimal strategies exist. However, countably additive strategies are not pessimistic optimal, and also not every pure charge is pessimistic optimal.

Consider the following binary game. Let $A_{1}=A_{2}=\mathbb{N}$ and define the payoff function $u$ by

$$
u(m, n)= \begin{cases}0 & \text { if } m=n \\ 1 & \text { otherwise }\end{cases}
$$

The payoffs are displayed in the following figure.

\begin{tabular}{|c|cccc|}
\hline$u$ & 1 & 2 & 3 & $\ldots$ \\
\hline 1 & 0 & 1 & 1 & $\ldots$ \\
2 & 1 & 0 & 1 & $\ldots$ \\
3 & 1 & 1 & 0 & $\ldots$ \\
$\vdots$ & $\vdots$ & $\vdots$ & $\vdots$ & $\ddots$ \\
\hline
\end{tabular}

In this game player 1 has to hide at a certain place $m \in \mathbb{N}$. Player 2 wins if he searches at the place where player 1 is hidden, otherwise player 1 wins.

First we argue that $v_{1}^{p}=1$. Take $k \in \mathbb{N}$ and consider the countably additive strategy $p_{k}$ for player 1 such that

$$
p_{k}(m)= \begin{cases}\frac{1}{k} & \text { if } m \leq k \\ 0 & \text { otherwise }\end{cases}
$$

It is clear that $U\left(<p_{k}, \mu_{2}>\right) \geq \frac{k-1}{k}$ for every $\mu_{2} \in \mathcal{C}(\mathbb{N})$. Therefore $v_{1}^{p}=1$ indeed.

Now notice that no strategy $p_{1} \in \Delta(\mathbb{N})$ can be pessimistic optimal for player 1 . There exists $n \in \mathbb{N}$ such that $p_{1}(n)>0$, so if player 2 plays the Dirac measure $\delta(n)$, then $U\left(<p_{1}, \delta(n)>\right)<1$. Also notice that a pure charge $\mu \in \mathcal{Q}(\mathbb{N})$ with $\mu(B) \in\{0,1\}$ for all $B \subseteq \mathbb{N}$ is not pessimistic optimal either, since if player 2 also plays $\mu$, then $U^{p}(<\mu, \mu>)=0$.

Now we define the strategy $\kappa_{1}$ for player 1 and show that it is pessimistic optimal. For $k \in \mathbb{N}$, and $\ell \in \mathbb{N}$ with $1 \leq \ell \leq 2^{k}$, define

$$
E_{\ell}^{k}=\left\{2^{k} \cdot n+\ell \mid n \in \mathbb{N} \cup\{0\}\right\} .
$$

There exists a pure charge $\kappa_{1}$ with the property that, for all $k$ and $\ell, \kappa_{1}\left(E_{\ell}^{k}\right)=\left(\frac{1}{2}\right)^{k} \cdot{ }^{13}$ Suppose player 1 plays charge $\kappa_{1}$. Let $\mu_{2}$ be any charge for player 2 .

One possible cover of the diagonal is

$$
(E \times E) \cup(O \times O),
$$

where $E$ is the set of even numbers and $O$ is the set of odd numbers. This cover has a probability of $\frac{1}{2} \cdot \mu_{2}(E)+\frac{1}{2} \cdot \mu_{2}(O)$ $=\frac{1}{2}$. In general, for fixed $k$,

$$
\bigcup_{\ell=1}^{2^{k}}\left(E_{\ell}^{k} \times E_{\ell}^{k}\right)
$$

covers the diagonal. The probability of this cover is

$$
\sum_{\ell=1}^{2^{k}}\left(\frac{1}{2}\right)^{k} \cdot \mu_{2}\left(E_{\ell}^{k}\right)=\left(\frac{1}{2}\right)^{k} .
$$

13 Since for each $k$ the collection of sets $E_{\ell}^{k}$ is a partition, it is straightforward to define $\kappa_{1}$ on the smallest field containing all singletons and all sets $E_{\ell}^{k}$. The existence of such a charge $\kappa_{1}$ defined on all subsets of $\mathbb{N}$ then follows from Theorem C.3. 
Thus, the infimum of the probabilities of these covers of the diagonal is 0 . This implies that $U^{p}\left(<\kappa_{1}, \mu_{2}>\right)=1$. Thus, $\kappa_{1}$ is pessimistic optimal for player 1 .

As a final remark, note that, since $v_{1}^{p}=1$, also $v_{1}^{o}=1$. Then, just like in the Wald game, the set $\mathcal{Q}(\mathbb{N})$ of all pure probability charges is the set of optimistic optimal strategies for player $1 .^{14}$

We do not know whether pessimistic optimal strategies always exist. For showing that optimistic optimal strategies exist, both in Theorem 5.1 and in Remark 5.1 we used an argument based on double integrals. More precisely, in both cases we used the fact that the optimistic payoff for player 1 is the same or higher than his payoff calculated through double integrals. The same argument cannot be used for pessimistic optimal strategies. We found games where pessimistic optimal strategies do exist, but we do not know whether this is the case in general for all games.

\subsection{Optimality for double integrals}

When player 1 (player 2) uses $U^{21}$ to evaluate outcomes, we say that an optimal strategy for player 1 (player 2 ) is 21-optimal. The notion of 12-optimality can be defined similarly.

Theorem 5.2. Player 2 has 21-optimal strategies. Player 1 has 12-optimal strategies.

Proof. The first half of the assertion is an immediate consequence of Lemma B.1 and the fact from Theorem 4.1 that $v_{1}^{f}=v_{2}^{21}$. The proof of the second half is similar.

Proposition 5.3. Let $\kappa_{1}$ be a pessimistic optimal strategy for player 1. Then it is an 21-optimal strategy for player 1. Similarly, if a strategy is pessimistic optimal for player 2, then it is also 12-optimal for player 2.

Proof. The first half of the assertion is an immediate consequence of Theorem 4.1 and the fact that $U^{p} \leq U^{21}$. The proof of the second half is similar.

\section{Zero-sum games with charges}

When both players take the same extension function, the resulting game with charges is also zero-sum. In this section we analyze when-meaning for which types of extensions-such a resulting game has a value. The following two results are both direct consequences of Theorem 4.1 .

Corollary 6.1. Suppose that one player is optimistic, and that the other player is pessimistic. Then every resulting zero-sum game with charges has a value.

Corollary 6.2. Suppose that player 1 evaluates via $U^{21}$ and that player 2 evaluates via $-U^{21}$. Then every resulting zero-sum game with charges has a value. Similarly, there is a value, when player 1 evaluates via $U^{12}$ and player 2 evaluates via $-U^{12}$.

Thus, when the players agree on a given order of integration as an expected payoff, for example $U^{21}$, then any zero-sum game with charges has a value. This is well-known in the literature. Heath and Sudderth (1972) also show in Theorem 3 that $v_{1}^{21}=v_{2}^{21}$.

It is tempting to think that, whenever the players agree on the extension and consequently the resulting game is zerosum, this game automatically has a value. However, the next example shows this is not true.

Example 6.1. Even when the players agree on the extension function, and the resulting game with charges is zero-sum, the value of the game need not exist. Again consider the Wald game from Example 3.1 with payoff matrix

\begin{tabular}{|c|cccc|}
\hline$u$ & 1 & 2 & 3 & $\ldots$ \\
\hline 1 & 1 & 0 & 0 & $\ldots$ \\
2 & 1 & 1 & 0 & $\ldots$ \\
3 & 1 & 1 & 1 & $\ldots$ \\
$\vdots$ & $\vdots$ & $\vdots$ & $\vdots$ & $\ddots$ \\
\hline
\end{tabular}

\footnotetext{
14 Also Capraro and Scarsini (2013) analyze this game in Example 6.9. in their paper. Even though they consider different payoff extensions, they also find that the set of optimal strategies equals the set of pure charges for this game. Additionally, Pivato (2014) in subsection 4.2 looks at a game with the same payoff function on pure action profiles, but the players' mixed strategies are nonstandard probabilities. He shows in Proposition 8 that if each player plays a uniform distribution, then this strategy profile is a Nash-equilibrium.
} 
We construct a total ordering on the set $\mathcal{C}(\mathbb{N})$ of probability charges on $\mathbb{N}$. Take a countable sequence $\left(\mu_{k}\right)_{k \in \mathbb{N}}$ of pure probability charges where $\mu_{k} \neq \mu_{n}$ if $k \neq n$, and write $Q=\left\{\mu_{k} \mid k \in \mathbb{N}\right\}$. By Axiom of Choice, we can take a total ordering $\succeq$ of the set $\mathcal{C}(\mathbb{N}) \backslash Q$ of the remaining probability charges on $\mathbb{N}$. This total ordering can be extended to the set $\mathcal{C}(\mathbb{N})$ of probability charges on $\mathbb{N}$ as follows. For the elements of the sequence, if $n>k$, then $\mu_{n} \succ \mu_{k}$. For any $v \in \mathcal{C}(\mathbb{N}) \backslash Q$ and for any $n \in \mathbb{N}$ we define $\mu_{n} \succ v$. Hence, the total ordering $\succeq$ of the set $\mathcal{C}(\mathbb{N})$ is such that, for every probability charge $v \in \mathcal{C}(\mathbb{N})$, there is a pure probability charge $\mu \in Q$ with $\mu \succ v$.

Next, for any pair of probability charges $\left(\mu_{1}, \mu_{2}\right)$ we define an extension $\tau\left(\mu_{1}, \mu_{2}\right)$ by letting for all $K \subseteq \mathbb{N}$

$$
\tau\left(\mu_{1}, \mu_{2}\right)(K)= \begin{cases}\int_{n \in \mathbb{N}} \int_{m \in \mathbb{N}} \mathbb{I}_{K}(m, n) d \mu_{1} d \mu_{2} & \text { if } \mu_{1} \succeq \mu_{2} \\ \int_{m \in \mathbb{N}} \int_{n \in \mathbb{N}} \mathbb{I}_{K}(m, n) d \mu_{2} d \mu_{1} & \text { if } \mu_{2} \succ \mu_{1} .\end{cases}
$$

Note that $\tau\left(\mu_{1}, \mu_{2}\right)$ is indeed an extension of $\left(\mu_{1}, \mu_{2}\right)$. When we apply this extension function to the Wald game, by Lemma 3.2 the resulting zero-sum game on charges is

$$
U\left(\mu_{1}, \mu_{2}\right)= \begin{cases}U^{12}\left(\mu_{1}, \mu_{2}\right) & \text { if } \mu_{1} \succeq \mu_{2} \\ U^{21}\left(\mu_{1}, \mu_{2}\right) & \text { if } \mu_{2} \succ \mu_{1} .\end{cases}
$$

Intuitively, if $\mu_{1}$ is equal to $\mu_{2}$ or $\mu_{1}$ is enumerated later than $\mu_{2}$ by $\succeq$, then the inner integral is taken with respect the strategy of player 1 . As we know, this favors player 1 in the Wald game. Otherwise, the inner integral is taken with respect to the strategy of player 2 .

Take any $\mu_{1} \in \mathcal{C}(\mathbb{N})$. Then, by our construction of the total ordering $\succeq$, there is a pure charge $\mu_{2}$ such that $\mu_{2} \succ \mu_{1}$. Then $U^{\tau}\left(\mu_{1}, \mu_{2}\right)=0$. So, $\sup _{\mu_{1} \in \mathcal{C}(\mathbb{N})} \inf _{\mu_{2} \in \mathcal{C}(\mathbb{N})} U^{\tau}\left(\mu_{1}, \mu_{2}\right)=0$. Similarly, one can show that $\inf _{\mu_{2} \in \mathcal{C}(\mathbb{N})} \sup _{\mu_{1} \in \mathcal{C}(\mathbb{N})} U^{\tau}\left(\mu_{1}, \mu_{2}\right)=$ 1 .

Thus there exists an extension $\tau\left(\mu_{1}, \mu_{2}\right)$ for each pair of probability charges $\left(\mu_{1}, \mu_{2}\right)$ with

$$
\sup _{\mu_{1} \in \mathcal{C}(\mathbb{N})} \inf _{\mu_{2} \in \mathcal{C}(\mathbb{N})} U^{\tau}\left(\mu_{1}, \mu_{2}\right)<\inf _{\mu_{2} \in \mathcal{C}(\mathbb{N})} \sup _{\mu_{1} \in \mathcal{C}(\mathbb{N})} U^{\tau}\left(\mu_{1}, \mu_{2}\right) .
$$

So, the supremum and the infimum are not interchangeable for $\tau$, and the value $v^{\tau}$ does not exist. $\triangleleft$

Example 6.2. On the other hand, when both players are optimistic, by Theorem 5.1 both players have optimal strategies. However, the resulting game need not be a zero-sum game. For example, again consider the Wald game from Example 3.1. Clearly, for every $p_{1} \in \Delta(\mathbb{N})$ it holds that

$$
\inf _{n \in \mathbb{N}} u\left(<p_{1}, n>\right)=0 \text {. }
$$

Hence, $v_{1}=0$. We obtain similarly that $v_{2}=1 . \triangleleft$

\section{Concluding remarks}

We studied the effect of using finitely additive probability distributions, charges, in the context of zero-sum games. Due to the ambiguity associated with extending strategy profiles to charges on the space of pure action profiles, the use of charges gives rise to several methods to define the guarantee level of a player.

Specifically, we studied optimistic and pessimistic guarantee levels, and guarantee levels associated with double integrals. We showed that the pessimistic guarantee level for player 1, the optimistic guarantee level for player 2, and the guarantee levels for both players in which we first integrate with respect to the strategy of the 2nd player and then with respect to the 1st player, all coincide. Moreover, these guarantee levels can be calculated by computing the finitistic guarantee levels.

Thus, when player 1 is optimistic, and player 2 is pessimistic, any resulting (zero-sum) game has a value. The same holds when both players use the same order of integration for the approach based on double integrals. We also proved that players always have optimal strategies with respect to the optimistic guarantee level.

In conclusion, charges are useful to define the value of an arbitrary zero-sum game, and optimistic players always have optimal strategies. It is still an open question whether pessimistic players always have optimal strategies.

\section{Appendix A. Tools}

Lemma A.1. Let $X$ be a non-empty set. Let $F$ be the set of all bounded functions $f: X \rightarrow \mathbb{R}$. Let $S$ be the set of all simple functions in $F$. Let $\phi: F \rightarrow \mathbb{R}$ be a mapping such that for every $f \in F$

$$
\phi(f)=\sup \{\phi(s): s \in S \text { and } s \leq f\} .
$$

Let $\psi: F \rightarrow \mathbb{R}$ be linear and monotone. Suppose that $\phi(s)=\psi(s)$ for all $s \in S$. Then $\phi=\psi$. 
Proof. For every $s \in S$ and $f \in F$ with $s \leq f$ we have $\phi(s)=\psi(s) \leq \psi(f)$. Hence, for every $f \in F$

$$
\phi(f)=\sup \{\phi(s): s \in S \text { and } s \leq f\} \leq \psi(f) .
$$

Take $f \in F$. We prove $\phi(f) \geq \psi(f)$. Take $\varepsilon>0$. It is easy to see that there exists a simple function $s$ such that $s \leq f \leq$ $s+\varepsilon .{ }^{15}$ Thus

$$
\psi(f) \leq \psi(s+\varepsilon)=\psi(s)+\psi(\varepsilon)=\phi(s)+\varepsilon \psi(1) \leq \phi(f)+\varepsilon \psi(1) .
$$

Since $\varepsilon>0$ is arbitrary, $\psi(f) \leq \phi(f)$.

Lemma A.2. Let $X$ be a non-empty set. Let I be a set of indices. For each $k \in I$, let $\mu_{k}$ be a probability charge on X. Let $\tau$ be a probability charge on I. Consider the probability charge $\sigma$ on $X$ defined by

$$
\sigma(E)=\int_{k \in I} \mu_{k}(E) d \tau \quad \text { for all } E \subseteq X .
$$

Then, for any bounded function $f: X \rightarrow \mathbb{R}$,

$$
\int_{X} f(x) d \sigma=\int_{k \in I} \int_{X} f(x) d \mu_{k} d \tau .
$$

Proof. For any simple function $s: X \rightarrow \mathbb{R}$, where $s=\sum_{i=1}^{m} c_{i} \mathbb{I}_{F_{i}}$, we have

$$
\begin{aligned}
\int_{X} s(x) d \sigma & =\sum_{i=1}^{m} c_{i} \cdot \sigma\left(F_{i}\right)=\sum_{i=1}^{m} c_{i} \int_{k \in I} \mu_{k}\left(F_{i}\right) d \tau \\
& =\int_{k \in I} \sum_{i=1}^{m} c_{i} \mu_{k}\left(F_{i}\right) d \tau \\
& =\int_{k \in I} \int_{X} s(x) d \mu_{k} d \tau,
\end{aligned}
$$

where the first equality is based on the definition of the integral of a simple function and the second equality on the definition of $\sigma$. In view of Lemma A.1, taking $\phi(f)=\int_{X} f(x) d \sigma$ and $\psi(f)=\int_{k \in I} \int_{X} f(x) d \mu_{k} d \tau$, the proof is complete.

For a set $A_{1}$, let $\ell\left(A_{1}\right)$ be the collection of probability charges $p_{1} \in \mathcal{C}\left(A_{1}\right)$ for which there is a sequence of points $a_{1}^{1}, a_{1}^{2}, \ldots$ in $A_{1}$ and non-negative real numbers $c^{1}, c^{2}, \ldots$ such that $p_{1}=\sum_{k} c^{k} \cdot \delta\left(a_{1}^{k}\right)$, where $\delta\left(a_{1}^{k}\right)$ denotes the Dirac measure on $a_{1}^{k}$. Note that automatically $\sum_{k} c^{k}=1$. We assume that all points $a_{1}^{k}$ are different.

Lemma A.3. Let $p_{1} \in \ell\left(A_{1}\right)$ and $\mu_{2} \in \mathcal{C}\left(A_{2}\right)$. Then there is a unique $\mu \in \mathcal{C}\left(A_{1} \times A_{2}\right)$ such that $\mu \in<p_{1}, \mu_{2}>$. Consequently, the Fubini-equality (cf. Remark 3.1) holds for the pair $\left(p_{1}, \mu_{2}\right)$ and any bounded payoff function.

Proof. By Proposition C.5, we know that $<p_{1}, \mu_{2}>$ is not empty. Take $\mu \in<p_{1}, \mu_{2}>$ and $\kappa \in<p_{1}$, $\mu_{2}>$. Take a set $W \subseteq A_{1} \times A_{2}$. We show that $\mu(W)=\kappa(W)$.

A. We say that a set $R \subseteq A_{1} \times A_{2}$ is a rectangle if there are $B_{1} \subseteq A_{1}$ and $B_{2} \subseteq A_{2}$ such that $R=B_{1} \times B_{2}$. Observe that $\mu(R)=p_{1}\left(B_{1}\right) \cdot \mu_{2}\left(B_{2}\right)=\kappa(R)$ for all rectangles $R$.

B. Take any $\varepsilon>0$. Take $K \in \mathbb{N}$ such that $\sum_{k>K} c^{k} \leq \varepsilon$. For $k \leq K$, define

$$
B^{k}=\left\{\left(a_{1}, a_{2}\right) \in W \mid a_{1}=a_{1}^{k}\right\} \quad \text { and } \quad B=\bigcup_{k \leq K} B^{k} \text {. }
$$

Notice that each $B^{k}$ is a rectangle. So, $\mu\left(B^{k}\right)=\kappa\left(B^{k}\right)$ for each $k$. Further, since all points $a_{1}^{k}$ are different, $B$ is a finite union of disjoint rectangles $B^{k}, k \leq K$. So, since $\mu$ and $\kappa$ are finitely additive, we also have $\mu(B)=\kappa(B)$.

\footnotetext{
15 See p. 272 in the appendix by WD Sudderth, D Gilat and R Purves in Dubins et al. (2014). Indeed, consider the inverse images $f^{-1}[z \varepsilon,(z+1) \varepsilon)$, where $z$ is an integer. Since $f$ is bounded, only finitely many of them are non-empty. If $f^{-1}[z \varepsilon,(z+1) \varepsilon)$ is non-empty, then let $s$ take value $z \varepsilon$ on this set. It follows that $s \leq f \leq s+\varepsilon$.
} 
C. Also define

$$
Z_{1}=A_{1} \backslash\left\{a_{1}^{k} \in A_{1} \mid k \leq K\right\}
$$

and $Z=Z_{1} \times A_{2}$. Then $Z$ is a rectangle, so that $\mu(Z)=p_{1}\left(Z_{1}\right) \cdot \mu_{2}\left(A_{2}\right) \leq \varepsilon \cdot 1=\varepsilon$. In the same way we find $\kappa(Z) \leq \varepsilon$.

D. Now notice that $B \subseteq W \subseteq B \cup Z$, and $B$ and $Z$ are disjoint. So, by the additivity and monotonicity of $\mu, \mu(B) \leq$ $\mu(W) \leq \mu(B)+\mu(Z) \leq \mu(B)+\varepsilon$. Hence, since $\kappa(B)=\mu(B), \kappa(B) \leq \mu(W) \leq \kappa(B)+\varepsilon$. However, also $\kappa(B) \leq \kappa(W) \leq$ $\kappa(B)+\varepsilon$. It follows that $\mu(W)=\kappa(W)$.

\section{Appendix B. Proof of Theorem 4.1}

Lemma B.1. Player 2 has a strategy $\sigma_{2} \in \mathcal{C}\left(A_{2}\right)$ such that for every $\mu_{1} \in \mathcal{C}\left(A_{1}\right)$ we have

$$
U^{21}\left(\mu_{1}, \sigma_{2}\right) \leq v_{1}^{f} .
$$

Player 1 has a similar strategy $\sigma_{1}$.

Proof. A. Take $F_{1} \in \mathcal{F}\left(A_{1}\right)$. Take $k \in \mathbb{N}$. By definition of $v_{1}^{f}$ there is a set $F_{2 k} \in \mathcal{F}\left(A_{2}\right)$ with

$$
v\left(F_{1}, F_{2 k}\right) \leq v_{1}^{f}+\frac{1}{k} .
$$

Let $p_{2 k} \in \Delta\left(F_{2 k}\right)$ be an optimal strategy for player 2 in the game $G\left(F_{1}, F_{2 k}\right)$. Naturally, $p_{2 k}$ can also be seen as an element of $\mathcal{C}\left(A_{2}\right)$.

B. Let $\lambda$ be any pure probability charge on $\mathbb{N}$. Define a strategy $\sigma_{2, F_{1}} \in \mathcal{C}\left(A_{2}\right)$ for player 2 by

$$
\sigma_{2, F_{1}}(B)=\int_{k \in \mathbb{N}} p_{2 k}(B) d \lambda \quad \text { for all } B \subseteq A_{2} .
$$

C. For each $m \in F_{1}$, let $\delta(m)$ denote the Dirac measure on $m$. We have

$$
\begin{aligned}
U\left(<\delta(m), \sigma_{2, F_{1}}>\right) & =\int_{n \in A_{2}} u(m, n) d \sigma_{2, F_{1}}=\int_{k \in \mathbb{N}} \int_{n \in A_{2}} u(m, n) d p_{2 k} d \lambda \\
& \leq \int_{k \in \mathbb{N}}\left(v_{1}^{f}+\frac{1}{k}\right) d \lambda=v_{1}^{f} .
\end{aligned}
$$

Here the second equality follows from Lemma A.2 and the definition of $\sigma_{2, F_{1}}$ in part B. The inequality comes from the definition of $p_{2 k}$ in part $A$. The last equality is a consequence of the fact that $\lambda(\{1, \ldots, K\})=0$ for every $K \in \mathbb{N}$.

D. Let $\tau$ be a probability charge as in Lemma C.4 on $\mathcal{F}\left(A_{1}\right)$. Thus, for every $m \in A_{1}$ we have

$$
\tau\left(\left\{F_{1} \in \mathcal{F}\left(A_{1}\right) \mid m \in F_{1}\right\}\right)=1 .
$$

Define a strategy $\sigma_{2} \in \mathcal{C}\left(A_{2}\right)$ for player 2 by

$$
\sigma_{2}(B)=\int_{F_{1} \in \mathcal{F}\left(A_{1}\right)} \sigma_{2, F_{1}}(B) d \tau \quad \text { for all } B \subseteq A_{2} .
$$

E. We show that for every $\mu_{1} \in \mathcal{C}\left(A_{1}\right)$

$$
U^{21}\left(\mu_{1}, \sigma_{2}\right) \leq v_{1}^{f} .
$$

For every $m \in A_{1}$ we have

$$
\begin{aligned}
\int_{n \in A_{2}} u(m, n) d \sigma_{2} & =\int_{F_{1} \in \mathcal{F}_{1}} \int_{n \in A_{2}} u(m, n) d \sigma_{2, F_{1}} d \tau=\int_{F_{1} \in \mathcal{F}_{1}} U\left(<\delta(m), \sigma_{2, F_{1}}>\right) d \tau \\
& =\int_{F_{1} \in\left\{F \in \mathcal{F}_{1} \mid m \in F\right\}} U\left(<\delta(m), \sigma_{2, F_{1}}>\right) d \tau \leq v_{1}^{f} .
\end{aligned}
$$

Here the first equality follows from Lemma A.2 and the definition of $\sigma_{2}$ in part D. The third equality holds due to our choice of $\tau$ in part D. In the final step we can use inequality (B.1) from part A, since now $m \in F_{1}$. 
F. Since an integral is monotonic, for any $\mu_{1} \in \mathcal{C}\left(A_{1}\right)$

$$
U^{21}\left(\mu_{1}, \sigma_{2}\right)=\int_{m \in A_{1}} \int_{n \in A_{2}} u(m, n) d \sigma_{2} d \mu_{1} \leq \int_{m \in A_{1}} v_{1}^{f} d \mu_{1}=v_{1}^{f} .
$$

This concludes the proof.

Theorem B.2. Let $u$ be any zero-sum game. The guarantee levels of the players satisfy

$$
v_{1}^{f}=v_{1}^{d}=v_{1}^{p}=v_{2}^{o}=v_{1}^{21}=v_{2}^{21} \quad \text { and } \quad v_{2}^{f}=v_{2}^{d}=v_{2}^{p}=v_{1}^{o}=v_{2}^{12}=v_{1}^{12} .
$$

Moreover, if $\sigma_{1}$ and $\sigma_{2}$ are strategies as in Lemma B.1, then $\sigma_{1}$ is optimistic optimal for player 1, and $\sigma_{2}$ is optimistic optimal for player 2.

Proof. First we show that $v_{1}^{p} \leq v_{2}^{o} \leq v_{1}^{f}$. Then we show that $v_{1}^{f} \leq v_{1}^{d} \leq v_{1}^{p} \leq v_{1}^{21} \leq v_{2}^{21} \leq v_{1}^{f}$, and that $\sigma_{2}$ is optimistic optimal for player 2.

A. We show that $v_{1}^{p} \leq v_{2}^{o}$. It is clear from the definitions that $v_{1}^{p} \leq v_{2}^{o}$.

B. We show that $v_{2}^{o} \leq v_{1}^{f}$. Let $\sigma_{2} \in \mathcal{C}\left(A_{2}\right)$ be a strategy for player 2 as in Lemma B.1. Then, for every $\mu_{1} \in \mathcal{C}\left(A_{1}\right)$ we have

$$
U^{p}\left(\mu_{1}, \sigma_{2}\right) \leq U^{21}\left(\mu_{1}, \sigma_{2}\right) \leq v_{1}^{f} .
$$

Therefore,

$$
v_{2}^{o}=\inf _{\mu_{2} \in \mathcal{C}\left(A_{2}\right)} \sup _{\mu_{1} \in \mathcal{C}\left(A_{1}\right)} U^{p}\left(\mu_{1}, \mu_{2}\right) \leq \sup _{\mu_{1} \in \mathcal{C}\left(A_{1}\right)} U^{p}\left(\mu_{1}, \sigma_{2}\right) \leq v_{1}^{f} .
$$

C. We show that $v_{1}^{f} \leq v_{1}^{d}$. Take $F_{1} \in \mathcal{F}\left(A_{1}\right)$. Then

$$
\begin{aligned}
\sup _{\mu_{1} \in \Delta\left(F_{1}\right)} \inf _{\mu_{2} \in \mathcal{D}\left(A_{2}\right)} U\left(<\mu_{1}, \mu_{2}>\right) & =\sup _{\mu_{1} \in \Delta\left(F_{1}\right)} \inf _{n \in A_{2}} U\left(<\mu_{1}, \delta(n)>\right) \\
& \geq \inf _{F_{2} \in \mathcal{F}_{2}} \sup _{\mu_{1} \in \Delta\left(F_{1}\right)} \inf _{n \in F_{2}} U\left(<\mu_{1}, \delta(n)>\right) \\
& =\inf _{F_{2} \in \mathcal{F}_{2}} w\left(F_{1}, F_{2}\right) .
\end{aligned}
$$

Hence, $v_{1}^{d} \geq v_{1}^{f}$.

D. We show that $v_{1}^{d} \leq v_{1}^{p}$. Take a $p_{1} \in \mathcal{D}\left(A_{1}\right)$ and a $\mu_{2} \in \mathcal{C}\left(A_{2}\right)$. Let $\delta(n)$ denote the Dirac measure on $n \in A_{2}$. Then

$$
U\left(<p_{1}, \mu_{2}>\right)=\int_{n \in A_{2}} U\left(<p_{1}, \delta(n)>\right) d \mu_{2} \geq \inf _{n \in A_{2}} U\left(<p_{1}, \delta(n)>\right) .
$$

We used the fact that, since $p_{1} \in \ell\left(A_{1}\right)$, the extension for $\left(p_{1}, \mu_{2}\right)$ is unique by Lemma A.3. So,

$$
\inf _{\mu_{2} \in \mathcal{C}\left(A_{1}\right)} U\left(<p_{1}, \mu_{2}>\right) \geq \inf _{n \in A_{2}} U\left(<p_{1}, \delta(n)>\right) \geq \inf _{p_{2} \in \mathcal{D}\left(A_{2}\right)} U\left(<p_{1}, p_{2}>\right) .
$$

It follows that

$$
\sup _{p_{1} \in \mathcal{D}\left(A_{1}\right)} \inf _{\mu_{2} \in \mathcal{C}\left(A_{2}\right)} U\left(<p_{1}, \mu_{2}>\right) \geq \sup _{p_{1} \in \mathcal{D}\left(A_{1}\right)} \inf _{p_{2} \in \mathcal{D}\left(A_{2}\right)} U\left(<p_{1}, p_{2}>\right)=v_{1}^{d} .
$$

So,

$$
v_{1}^{p}=\sup _{\mu_{1} \in \mathcal{C}\left(A_{1}\right)} \inf _{\mu_{2} \in \mathcal{C}\left(A_{2}\right)} U^{p}\left(\mu_{1}, \mu_{2}\right) \geq \sup _{p_{1} \in \mathcal{D}\left(A_{1}\right)} \inf _{\mu_{2} \in \mathcal{C}\left(A_{2}\right)} U^{p}\left(p_{1}, \mu_{2}\right) \geq v_{1}^{d}
$$

E. We show that $v_{1}^{p} \leq v_{1}^{21}$. Let $\mu_{1} \in \mathcal{C}\left(A_{1}\right)$ and $\mu_{2} \in \mathcal{C}\left(A_{2}\right)$ be two probability charges. We know from Lemma 3.2 that the probability charge $\kappa$ defined by

$$
\kappa(E)=\int_{m \in A_{1}} \int_{n \in A_{2}} \mathbb{I}_{E}(m, n) d \mu_{2} d \mu_{1} \quad \text { for all } E \subseteq \mathbb{N}
$$

is an extension of the strategy profile $\left(\mu_{1}, \mu_{2}\right)$, and that by definition $U^{21}\left(\mu_{1}, \mu_{2}\right)=U(\kappa)$. Hence, by definition of $U^{p}\left(\mu_{1}, \mu_{2}\right)$, we have $U^{p}\left(\mu_{1}, \mu_{2}\right) \leq U(\kappa)=U^{21}\left(\mu_{1}, \mu_{2}\right)$. So, 


$$
v_{1}^{p}=\sup _{\mu_{1} \in \mathcal{C}\left(A_{1}\right)} \inf _{\mu_{2} \in \mathcal{C}\left(A_{2}\right)} U^{p}\left(\mu_{1}, \mu_{2}\right) \leq \sup _{\mu_{1} \in \mathcal{C}\left(A_{1}\right)} \inf _{\mu_{2} \in \mathcal{C}\left(A_{2}\right)} U^{21}\left(\mu_{1}, \mu_{2}\right) \leq v_{1}^{21}
$$

F. We argue that $v_{1}^{21} \leq v_{2}^{21}$. It is clear from the definitions that $v_{1}^{21} \leq v_{2}^{21}$.

G. We argue that $v_{2}^{21} \leq v_{1}^{f}$. It follows from Lemma B.1 that $v_{2}^{21} \leq v_{1}^{f}$.

H. Hence, $v_{1}=v_{1}^{f}=v_{1}^{d}=v_{1}^{p}=v_{2}^{o}=v_{1}^{21}=v_{2}^{21}$. It now follows from Lemma B.1 that $\sigma_{2}$ is optimistic optimal for player 2.

\section{Appendix C. Existence of charges}

A collection $\mathcal{F}$ of subsets of a non-empty set $X$ is called a field if:

[1] $\phi \in \mathcal{F}$

[2] if $A \in \mathcal{F}$ then $X \backslash A \in \mathcal{F}$ and

[3] if $A_{1}, \ldots, A_{n}$ are elements of $\mathcal{F}$, then $\bigcup_{i=1}^{n} A_{i} \in \mathcal{F}$.

The following observation can be found as 1.(ii) on page 268 in Loś and Marczewski (1949).

Lemma C.1. Let $\mathcal{F}$ be a field on $X$, and let $E \subseteq X$ be given. There exists a smallest field $\mathcal{F}(E)$ that contains both $E$ and all elements of $\mathcal{F}$. Moreover,

$$
S \in \mathcal{F}(E) \Leftrightarrow \text { there are } A, B \in \mathcal{F} \text { with } S=(A \cap E) \cup(B \backslash E) .
$$

Proof. Suppose that $\mathcal{F} \neq \mathcal{P}(X)$ and $E \notin \mathcal{F}$. It is clear that a smallest field $\mathcal{F}(E)$ exists that contains both $E$ and all elements of $\mathcal{F}$. It is also clear that all elements of the form $(A \cap E) \cup(B \backslash E)$ with $A, B \in \mathcal{F}$ are contained in $\mathcal{F}(E)$.

Define the set $\mathcal{G}$ by

$$
S \in \mathcal{G} \Leftrightarrow \text { there are } A, B \in \mathcal{F} \text { with } S=(A \cap E) \cup(B \backslash E) .
$$

We show that $\mathcal{G}$ is a field that contains both $E$ and all elements of $\mathcal{F}$. Then $\mathcal{F}(E) \subseteq \mathcal{G}$, which concludes the proof.

We show that $\mathcal{F} \subseteq \mathcal{G}$ and $E \in \mathcal{G}$. Take $C \in \mathcal{F}$. Then, by choosing $A=B=C$, we see that $C \in \mathcal{G}$. So, $\mathcal{F} \subseteq \mathcal{G}$. Further, by choosing $A=X, B=\phi$, we see that $E \in \mathcal{G}$.

We show that $\mathcal{G}$ is a field. Clearly, $\phi \in \mathcal{G}$. Take $S \in \mathcal{G}$. Take $A, B \in \mathcal{F}$ with $S=(A \cap E) \cup(B \backslash E)$. Then $X \backslash S=\left(A^{c} \cap E\right) \cup$ $\left(B^{c} \backslash E\right.$ ). Since $\mathcal{F}$ is a field, we know that $A^{c}, B^{c} \in \mathcal{F}$, and we can conclude that $X \backslash S \in \mathcal{G}$.

Take $S_{1}, \ldots, S_{n} \in \mathcal{G}$. Take $A_{k}, B_{k} \in \mathcal{F}$ with $S_{k}=\left(A_{k} \cap E\right) \cup\left(B_{k} \backslash E\right)$. Then

$$
\bigcup_{k} S_{k}=\left[\left(\bigcup_{k} A_{k}\right) \cap E\right] \cup\left[\left(\bigcup_{k} B_{k}\right) \backslash E\right] \text {. }
$$

Since $\mathcal{F}$ is a field, $\bigcup_{k} A_{k}, \bigcup_{k} B_{k} \in \mathcal{F}$. Hence, $\bigcup_{k} S_{k} \in \mathcal{G}$.

A function $\mu: \mathcal{F} \rightarrow[0,1]$ is called a charge on $\mathcal{F}$ if $\mu(X)=1$, and $\mu(A \cup B)=\mu(A)+\mu(B)$ for all disjoint sets $A, B \in \mathcal{F}$. In particular $\mu(\phi)=0$ for any charge $\mu$.

Take $E \subseteq X$. A charge $v$ on $\mathcal{F}(E)$ is called an extension to $\mathcal{F}(E)$ of a charge $\mu$ on $\mathcal{F}$ if $\nu(A)=\mu(A)$ for all $A \in \mathcal{F}$. Take $Y \subset X$.

$$
\alpha(\mu)(Y)=\sup \{\mu(A) \mid A \in \mathcal{F}, A \subseteq Y\} \quad \text { and } \quad \beta(\mu)(Y)=\inf \{\mu(B) \mid B \in \mathcal{F}, Y \subseteq B\} .
$$

Clearly $\alpha(\mu)(Y) \leq \beta(\mu)(Y)$. The following observation, which we prove for completeness, follows from Theorem 2 in Lośs and Marczewski (1949).

Lemma C.2. Let $\mathcal{F}$ be a field, and let $\mu$ be a charge on $\mathcal{F}$. Let $E \in \mathcal{P}(X)$ and $r \in \mathbb{R}$ with $\alpha(\mu)(E) \leq r \leq \beta(\mu)(E)$. Then there exists an extension $v$ of $\mu$ to $\mathcal{F}(E)$ such that $v(E)=r$.

Proof. We show there exists a charge $v$ on $\mathcal{F}(E)$ with $\nu(A)=\mu(A)$ for all $A \in \mathcal{F}$ and $v(E)=r$.

A. We first define an extension $v$ with $v(E)=\alpha(\mu)(E)$. Define $v: \mathcal{F}(E) \rightarrow \mathbb{R}$ as follows. Take $S \in \mathcal{F}(E)$. Take $A, B \in \mathcal{F}$ with $S=(A \cap E) \cup(B \backslash E)$. Define

$$
\nu(S)=\alpha(\mu)(A \cap E)+\mu(B)-\alpha(\mu)(B \cap E) .
$$


A1. We show that this is a valid definition. Take $A^{\prime}, B^{\prime} \in \mathcal{F}$ with $S=\left(A^{\prime} \cap E\right) \cup\left(B^{\prime} \backslash E\right)$. Then $A \cap E=A^{\prime} \cap E$. So, clearly

$$
\alpha(\mu)(A \cap E)=\alpha(\mu)\left(A^{\prime} \cap E\right) .
$$

Also $B \backslash E=B^{\prime} \backslash E$. Write $H=B \backslash B^{\prime}$ and $J=B^{\prime} \backslash B$. Then $H, J \in \mathcal{F}$. So, $\mu(B)-\mu(H)=\mu\left(B \cap B^{\prime}\right)=\mu\left(B^{\prime}\right)-\mu(J)$. Hence, it remains to show that

$$
\alpha(\mu)(B \cap E)-\mu(H)=\alpha(\mu)\left(B^{\prime} \cap E\right)-\mu(J) .
$$

Take $D \in \mathcal{F}$ with $D \subseteq B \cap E$. Write $G=D \backslash H$. Then $G \cup J \in \mathcal{F}$, and $G \cup J \subseteq B^{\prime} \cap E$. So,

$$
\begin{aligned}
\mu(D)-\mu(H) & \leq \mu(G \cup H)-\mu(H) \\
& =\mu(G)+\mu(H)-\mu(H) \\
& =\mu(G)+\mu(J)-\mu(J) \\
& =\mu(G \cup J)-\mu(J) \\
& \leq \alpha(\mu)\left(B^{\prime} \cap E\right)-\mu(J) .
\end{aligned}
$$

This shows that

$$
\alpha(\mu)(B \cap E)-\mu(H) \leq \alpha(\mu)\left(B^{\prime} \cap E\right)-\mu(J) .
$$

The reverse inequality follows similarly. This shows that the definition of $v$ is sound.

A2. Take $F \in \mathcal{F}$. We show that $\nu(F)=\mu(F)$. Take $A=B=F$. Then

$$
v(F)=\alpha(\mu)(A \cap E)+\mu(B)-\alpha(\mu)(B \cap E)=\mu(B)=\mu(F) .
$$

We show that $\nu(E)=\alpha(\mu)(E)$. Take $A=X$ and $B=\phi$. Then

$$
v(E)=\alpha(\mu)(X \cap E)+\mu(\phi)-\alpha(\mu)(\phi \cap E)=\alpha(\mu)(E) .
$$

A3. Take two sets $A$ and $B$ in $\mathcal{F}$ for which $A \cap E$ and $B \cap E$ are disjoint. We show that $\alpha(\mu)(A \cap E)+\alpha(\mu)(B \cap E)=$ $\alpha(\mu)((A \cup B) \cap E)$. It is clear that $\alpha(\mu)(A \cap E)+\alpha(\mu)(B \cap E) \leq \alpha(\mu)((A \cup B) \cap E)$. We show the reverse inequality. Take a $C \in \mathcal{F}$ with $C \subseteq(A \cup B) \cap E)$. Define $U=C \cap A$ and $V=C \cap B$. Then $U, V \in \mathcal{F}, U \cap V=\phi$, and $C=U \cup V$. So,

$$
\mu(C)=\mu(U)+\mu(V) \leq \alpha(\mu)(A \cap E)+\alpha(\mu)(B \cap E) .
$$

This completes the proof.

A4. We show that $v$ is additive on $\mathcal{F}(E)$. Take two disjoint sets $S$ and $T$ in $\mathcal{F}(E)$. We show that $v(S)+v(T)=v(S \cup T)$. Take sets $A, B, C, D \in \mathcal{F}$ such that $S=(A \cap E) \cup(B \backslash E)$ and $T=(C \cap E) \cup(D \backslash E)$. First note that

$$
S \cup T=((A \cup C) \cap E) \cup((B \cup D) \backslash E) .
$$

Note that $A \cap E$ and $C \cap E$ are disjoint. Moreover, $B$ and $D$ can be chosen in such a way that $B \cap D=\phi$. Then, using $\mathbf{A 3}$,

$$
\begin{aligned}
v(S)+v(T) & =\alpha(\mu)(A \cap E)+\mu(B)-\alpha(B \cap E)+\alpha(\mu)(C \cap E)+\mu(D)-\alpha(D \cap E) \\
& =\alpha(\mu)((A \cup C) \cap E)+\mu(B \cup D)-\alpha((B \cup D) \cap E) \\
& =v(S \cup T) .
\end{aligned}
$$

B. In the same way we can construct $\kappa$ on $\mathcal{G}$ with $\kappa(E)=\beta(\mu)(E)$. Taking a convex combination shows that we can find a $v$ on $\mathcal{G}$ with $v(E)=r$.

Let $\mathcal{P}(X)$ denote the collection of subsets of $X$. Note that $\mathcal{P}(X)$ is a field. Let $\mu$ be a charge on $\mathcal{F}$. An extension of $\mu$ is a charge $v$ on $\mathcal{P}(X)$ with $v(A)=\mu(A)$ for all $A \in \mathcal{F}$. Let $\mu$ be an extension of $\left(\mu_{1}, \mu_{2}\right)$. By monotonicity of $\mu$,

$$
\alpha(\mu)(E) \leq \mu(E) \leq \beta(\mu)(E)
$$

for every set $E \in \mathcal{P}(X \times X)$.

Theorem C.3. Assume Axiom of Choice. Let $\mathcal{F}$ be a field, and let $\mu$ be a charge on $\mathcal{F}$. Let $E \in \mathcal{P}(X)$ and $r \in \mathbb{R}$ with $\alpha(\mu)(E) \leq r \leq$ $\beta(\mu)(E)$. Then there exists an extension $\nu$ of $\mu$ such that $\nu(E)=r$.

Proof. The statement now follows from Lemma C.2 and the Lemma of Zorn. 
Corollary C.4. Let $\Omega$ be a non-empty set. Let $\mathcal{F}$ denote the collection of all finite subsets of $\Omega$. Then there is a probability charge $\tau$ on $\mathcal{F}$ such that for every $\omega \in \Omega$

$$
\tau(\{F \in \mathcal{F} \mid \omega \in F\})=1 .
$$

Proof. Take $F, G \in \mathcal{F}$. Define

$$
V[F, G]=\{H \in \mathcal{F} \mid F \subseteq H \text { and } G \cap H=\phi\} .
$$

Let $\mathcal{V}$ be the collection of all finite unions of such sets $V[F, G]$. This collection is a field. Moreover, define $\tau$ on $\mathcal{V}$ as the linear extension of

$$
\tau(V[F, G])= \begin{cases}1 & \text { if } G=\phi \\ 0 & \text { otherwise. }\end{cases}
$$

Then $\tau$ is a probability charge on $\mathcal{V}$. The result now follows directly from Theorem C.3.

For probability charges $\mu_{1} \in \mathcal{C}\left(A_{1}\right)$ and $\mu_{2} \in \mathcal{C}\left(A_{2}\right)$ and a set $E \in \mathcal{P}\left(A_{1} \times A_{2}\right)$, we define

$$
\alpha\left(\mu_{1}, \mu_{2}\right)(E)=\sup \left\{\sum_{i=1}^{k} \mu_{1}\left(C_{i}\right) \cdot \mu_{2}\left(D_{i}\right) \mid C_{i} \times D_{i} \text { are mutually disjoint, contained in } E\right\},
$$

and

$$
\beta\left(\mu_{1}, \mu_{2}\right)(E)=\inf \left\{\sum_{i=1}^{k} \mu_{1}\left(C_{i}\right) \cdot \mu_{2}\left(D_{i}\right) \mid C_{i} \times D_{i} \text { are mutually disjoint, and cover } E\right\} .
$$

Clearly, $\alpha\left(\mu_{1}, \mu_{2}\right)(E) \leq \beta\left(\mu_{1}, \mu_{2}\right)(E)$. Let $\mu$ be an extension of $\left(\mu_{1}, \mu_{2}\right)$. By monotonicity of $\mu$,

$$
\alpha\left(\mu_{1}, \mu_{2}\right)(E) \leq \mu(E) \leq \beta\left(\mu_{1}, \mu_{2}\right)(E)
$$

for every set $E \in \mathcal{P}\left(A_{1} \times A_{2}\right)$.

Proposition C.5. Let $\mu_{1} \in \mathcal{C}\left(A_{1}\right)$ and $\mu_{2} \in \mathcal{C}\left(A_{2}\right)$. Let $E \in \mathcal{P}\left(A_{1} \times A_{2}\right)$ and $r \in \mathbb{R}$ with $\alpha\left(\mu_{1}, \mu_{2}\right)(E) \leq r \leq \beta\left(\mu_{1}\right.$, $\left.\mu_{2}\right)(E)$. Then there exists an extension $\mu$ of $\left(\mu_{1}, \mu_{2}\right)$ such that $\mu(E)=r$.

\section{References}

Aliprantis, D., Border, K.C., 2005. Infinite Dimensional Analysis. Springer, Berlin.

Aumann, R.J., 1974. Subjectivity and correlation in randomized strategies. J. Math. Econ. 1 (1), 67-96.

Bingham, N.H., 2010. Finite additivity versus countable additivity. J. Électron. Hist. Probab. Stat. 6 (1).

Capraro, V., Scarsini, M., 2013. Existence of equilibria in countable games: an algebraic approach. Games Econ. Behav. 79 (C), $163-180$.

Dubins, L.E., Savage, L.J., 2014. In: How to Gamble If You Must: Inequalities for Stochastic Processes. Dover Publications, New York. Edited and updated by W.D. Sudderth and D. Gilat.

Dunford, N., Schwartz, J.T., 1964. Linear Operators, Part I: General Theory. Interscience Publishers, New York.

de Finetti, B., 1975. The Theory of Probability (2 Volumes). J. Wiley and Sons, Chichester.

Gilboa, I., Schmeidler, D., 1989. Maxmin expected utility with non-unique prior. J. Math. Econom. 18 (2), $141-153$.

Harris, J.H., Stinchcombe, M.B., Zame, W.R., 2005. Nearly compact and continuous normal form games: characterizations and equilibrium existence. Games Econ. Behav. 50, 208-224.

Heath, D., Sudderth, W., 1972. On a theorem of de Finetti, oddsmaking and game theory. Ann. Math. Stat. 43, 2072-2077.

Kindler, J., 1983. A general solution concept for two-person zero sum games. J. Optim. Theory Appl. 40, 105-119.

Lehrer, E., 2009. A new integral for capacities. Econ. Theory 39, 157-176.

Loś, J., Marczewski, E., 1949. Extensions of measure. Fundam. Math. 36, 267-276.

Maitra, A., Sudderth, W., 1993. Finitely additive and measurable stochastic games. Int. J. Game Theory 22, 201-223.

Maitra, A., Sudderth, W., 1998. Finitely additive stochastic games with Borel measurable payoffs. Int. J. Game Theory 27, 257-267.

Marinacci, M., 1997. Finitely additive and epsilon Nash equilibria. Int. J. Game Theory 26 (3), 315-333.

Pivato, M., 2014. Additive representation of separable preferences over infinite products. Theory Dec. 77 (1), $31-83$.

Rao, K.P.S.B., Rao, B., 1983. Theory of Charges: A Study of Finitely Additive Measures. Academic Press, New York.

Reny, P.J., 1999. On the existence of pure and mixed strategy Nash equilibria in discontinuous games. Econometrica 67 (5), $1029-1056$.

Savage, L.J., 1972. The Foundations of Statistics. Dover Publications, New York.

Schervish, M.J., Seidenfeld, T., 1996. A fair minimax theorem for two-person (zero-sum) games involving finitely additive strategies. In: Berry, D.A., Chaloner,

K.M., Geweke, J.K. (Eds.), Bayesian Analysis in Statistics and Econometrics. Wiley, New York, pp. 557-568.

Schirokauer, O., Kadane, J.B., 2007. Uniform distributions on the natural numbers. J. Theor. Probab. 20 (3), $429-441$.

Schmeidler, D., 1989. Subjective probability and expected utility without additivity. Econometrica 57, $571-587$.

Sudderth, W., 2016. Finitely additive dynamic programming. Math. Oper. Res. 41, 92-108.

von Neumann, J., 1928. Zur Theorie der Gesellschaftsspiele. Math. Ann. 100, 295-320.

Wald, A., 1945. Generalization of a theorem by v. Neumann concerning zero sum two person games. Ann. Math. 2 (46), $281-286$.

Wald, A., 1950. Statistical Decision Functions. Wiley, New York.

Yanovskaya, E.B., 1970. The solution of infinite zero-sum two-person games with finitely additive strategies. Theory Probab. Appl. 15 (1), 153-158. 